\title{
Paired Stimulation for Spike-Timing-Dependent Plasticity in Primate Sensorimotor Cortex
}

\author{
Stephanie C. Seeman, ${ }^{1,2} \oplus^{-}$Brian J. Mogen, ${ }^{1,2,3}$ @Eberhard E. Fetz, ${ }^{1,2,3}$ and $\oplus^{-S t e v e ~ I . ~ P e r l m u t t e r ~}{ }^{1,2}$ \\ ${ }^{1}$ Department of Physiology and Biophysics, Washington National Primate Research Center, ${ }^{2}$ Center for Sensorimotor Neural Engineering, and ${ }^{3}$ Department \\ of Bioengineering, University of Washington, Seattle, Washington 98915
}

Classic in vitro studies have described spike-timing-dependent plasticity (STDP) at a synapse: the connection from neuron A to neuron B is strengthened (or weakened) when A fires before (or after) B within an optimal time window. Accordingly, more recent in vivo works have demonstrated behavioral effects consistent with an STDP mechanism; however, many relied on single-unit recordings. The ability to modify cortical connections becomes useful in the context of injury, when connectivity and associated behavior are compromised. To avoid the need for long-term, stable isolation of single units, one could control timed activation of two cortical sites with paired electrical stimulation. We tested the hypothesis that STDP could be induced via prolonged paired stimulation as quantified by cortical evoked potentials (EPs) in the sensorimotor cortex of awake, behaving monkeys. Paired simulation between two interconnected sites produced robust effects in EPs consistent with STDP, but only at 2/15 tested pairs. The stimulation protocol often produced increases in global network excitability or depression of the conditioned pair. Together, these results suggest that paired stimulation in vivo is a viable method to induce STDP between cortical populations, but that factors beyond activation timing must be considered to produce conditioning effects.

Key words: connectivity; evoked potentials; free behavior

\section{Significance Statement}

Plasticity of neural connections is important for development, learning, memory, and recovery from injury. Cellular mechanisms underlying spike-timing-dependent plasticity have been studied extensively in vitro. Recent in vivo work has demonstrated results consistent with the previously defined cellular mechanisms; however, the output measure in these studies was typically an indirect assessment of plasticity at the neural level. Here, we show direct plasticity in recordings of neuronal populations in awake, behaving nonhuman primates induced by paired electrical stimulation. In contrast to in vitro studies, we found that plastic effects were only produced between specific cortical areas. These findings suggest that similar mechanisms drive plasticity in vitro and in vivo, but that cortical architecture may contribute significantly to site-dependent effects.

\section{Introduction}

Neuroplasticity underlies many brain functions from learning and memory to recovery from injury (Sanes and Donoghue, 2000; Stuchlik, 2014). Hebb postulated that the repeated associ-

Received June 27, 2016; revised Dec. 29, 2016; accepted Jan. 7, 2017.

Author contributions: S.C.S., B.J.M., E.E.F., and S.I.P. designed research; S.C.S. and B.J.M. performed research; S.C.S. and B.J.M. analyzed data; S.C.S., E.E.F., and S.I.P. wrote the paper.

This work was supported by the National Institutes of Health (Grant RR 00166; Grant NS12542 to E.E.F.; Grant NS088597 to S.I.P.; and Grant 5 T32 GM07108-40 to S.C.S) and the National Science Foundation (Grant EEC1028725). We thank L. Shupe for programming assistance and R. Schaefer and R. Robinson for assistance with animals.

The authors declare no competing financial interests.

Correspondence should be addressed to Steve I. Perlmutter, Department of Physiology and Biophysics, Washington National Primate Research Center, University of Washington, P.0. Box 357290, Seattle, WA 98195. E-mail: per@@uw.edu.

DOI:10.1523/JNEUROSCI.2046-16.2017

Copyright $\odot 2017$ the authors $\quad 0270-6474 / 17 / 371935-15 \$ 15.00 / 0$ ation of presynaptic and postsynaptic spiking modifies neuronal connections and forms the basis of learning (Hebb, 1949). The tenet of Hebbian plasticity as a basic mechanism of neuroplasticity has motivated an extensive field of research (Brown et al., 1990; Bi and Poo, 2001; Caporale and Dan, 2008).

Seminal in vitro studies by Markram et al. (1997) and Bi and Poo (1998) elucidated the activity-dependent nature by which a synapse is modified. If postsynaptic neuron B consistently generates an action potential within a short time window after receiving input from presynaptic neuron $\mathrm{A}$, then the connection from $A \rightarrow B$ is strengthened. If $B$ fires an action potential consistently before the input from $\mathrm{A}$, then the connection from $\mathrm{A} \rightarrow \mathrm{B}$ is weakened (Bi and Poo, 1998). Further studies have demonstrated the time course of this spike-timing-dependent plasticity (STDP) in many brain regions and organisms, including humans (Arai et al., 2011; Koch et al., 2013). However, intricacies of the precise temporal window and symmetry of the effect vary be- 
a

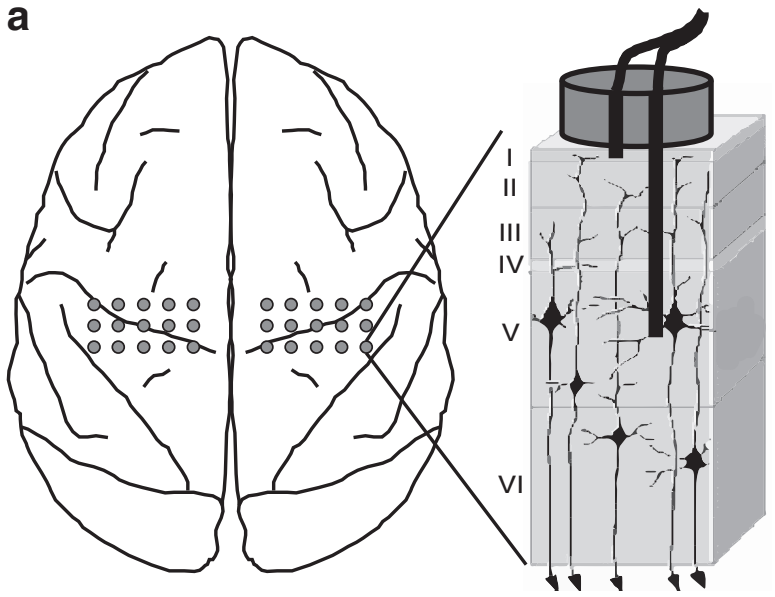

b

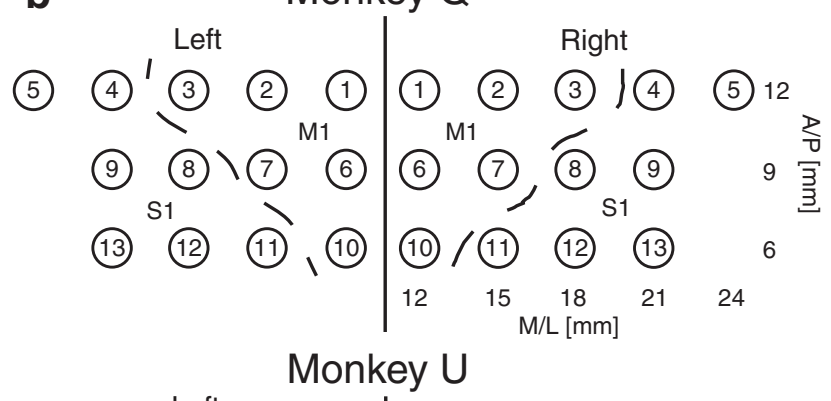

Figure 1. Implant schematic. $\boldsymbol{a}$, Top-down view of macaque brain showing the approximate position of each dual electrode with respect to midline and the central and arcuate sulci (gray circles). Expansion shows side view schematic of dual electrode leads with respect to cortical layers. $\boldsymbol{b}$, Numbered electrode sites for each monkey relative to the central sulcus (dotted line), as determined by median nerve stimulation.

tween type of synapse, brain areas, and species (Caporale and Dan, 2008; Feldman, 2012). Additional studies have shown that, although timing is important, other factors such as dendritic location (Froemke et al., 2005) and convergence of inputs (Sjöström et al., 2001), the relative timing of spike trains (Froemke and Dan, 2002), and background firing rate (Sjöström et al., 2001) all play a role on the effects of plasticity.

In vivo studies have demonstrated circuit and behavioral effects consistent with STDP using protocols such as pairing of sensory stimuli with electrical stimulation of central neurons or pairing of two different sensory stimuli (Feldman, 2012). Pairing of neural activity with electrical stimulation in primary motor cortex has also produced STDP-like changes in cortical connections in nonhuman primates (Jackson et al., 2006; Lucas and Fetz, 2013; Nishimura et al., 2013; Zanos, 2013) and rodents (Rebesco et al., 2010). In these studies, recorded spikes, muscle activity, or cortical field potentials were used to trigger stimulation repeatedly at a distant, but connected site. Varying the interval between the triggering event and stimulation produced an STDP time course remarkably similar to in vitro results.

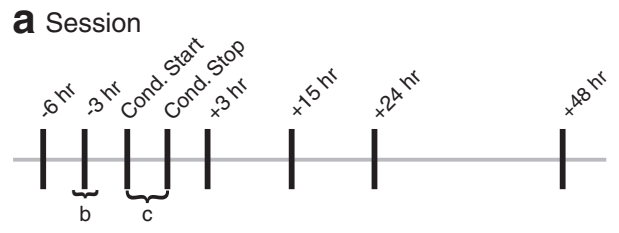

b Test Stimulation - in recording booth

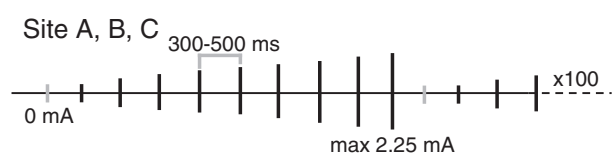

C Conditioning Stimulation - recording booth/home cage

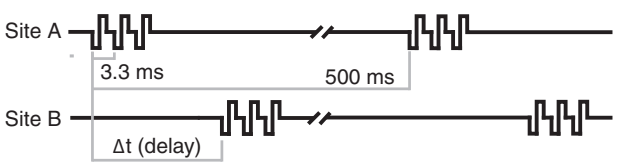

Figure 2. Experimental timeline. $\boldsymbol{a}$, Session timeline with all possible time points for testing and conditioning stimulation (not all time points were measured for every session). $\boldsymbol{b}$, Schematic of ramped test stimulation applied to sites A-C during each testing time point (e.g., " $b$ " in a). The exact number and magnitude of current steps varied across sessions, but was consistent within a session. C, Schematic of conditioning stimulation applied at sites $A$ and $B$ during conditioning period (e.g., "c" in $\boldsymbol{a}$ ).

These findings, coupled with recent advances in brain-computer interface technology, suggest that directed STDP could strengthen or reorganize spared connections preferentially and produce lasting, functional recovery after neural damage such as stroke or spinal cord injury (SCI). Functional motor recovery facilitated by STDP paradigms has been demonstrated in animal models of stroke (Guggenmos et al., 2013) and SCI (McPherson et al., 2015), as well as in stroke (Buetefisch et al., 2011) and SCI (Bunday and Perez, 2012) patients.

We sought to investigate the efficacy of open-loop, paired stimulation between sites in the sensorimotor cortex of awake, behaving monkeys to induce STDP. Although closed-loop stimulation has many advantages, one limitation is the need to record a strong and relevant trigger signal. This requirement is challenging for clinical applications that need to be effective over a patient's lifetime. Paired stimulation bypasses the need for recording a neural signal for activity-dependent stimulation. Rebesco and Miller (2011) showed that paired stimulation in awake, behaving rats can produce increased functional connectivity in cortex. We used paired stimulation at a fixed interval, which can be applied for any connected sites at any time, to control the timing of presynaptic and postsynaptic activity. In addition, previous findings have largely focused on changes in behavioral outputs in response to STDP protocols and the extent of cortical reorganization due to these protocols remains unclear. By recording LFPs from many sites in the hand area of sensorimotor cortex, we were able to measure direct changes in connectivity.

\section{Materials and Methods}

\section{Implant}

Dual electrodes. Two male monkeys (Macaca nemestrina), Q and U, were implanted bilaterally with custom-made dual electrodes (Fig. 1a) arranged in two $3 \times 5$ grids over sensorimotor cortex (Fig. 1b). Dual electrodes were constructed using two 0.005 -inch bare platinum-iridium (PtIr) wire rods cut to $3 \mathrm{~mm}$ (surface electrode) and $5 \mathrm{~mm}$ (intracortical electrode) and each soldered to 32 gauge, insulated lead wires (Fig. 1a). The connection between the PtIr rod and lead wire was further insulated with $10 \mu \mathrm{m}$ parylene by the University of Washington Microfabrication 


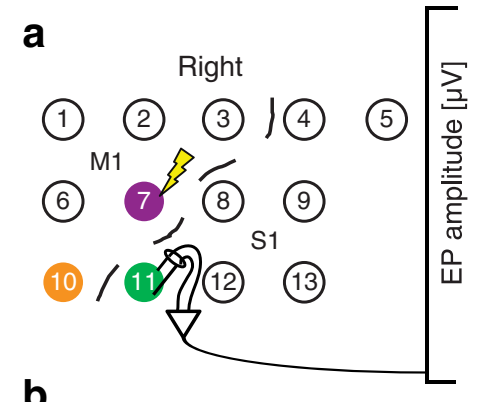

b
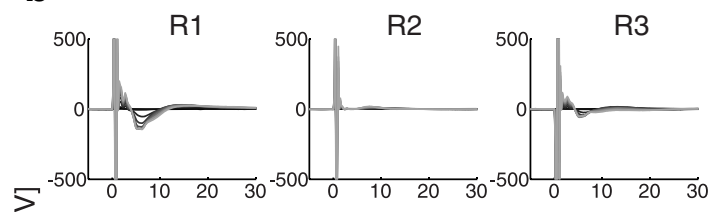

R3
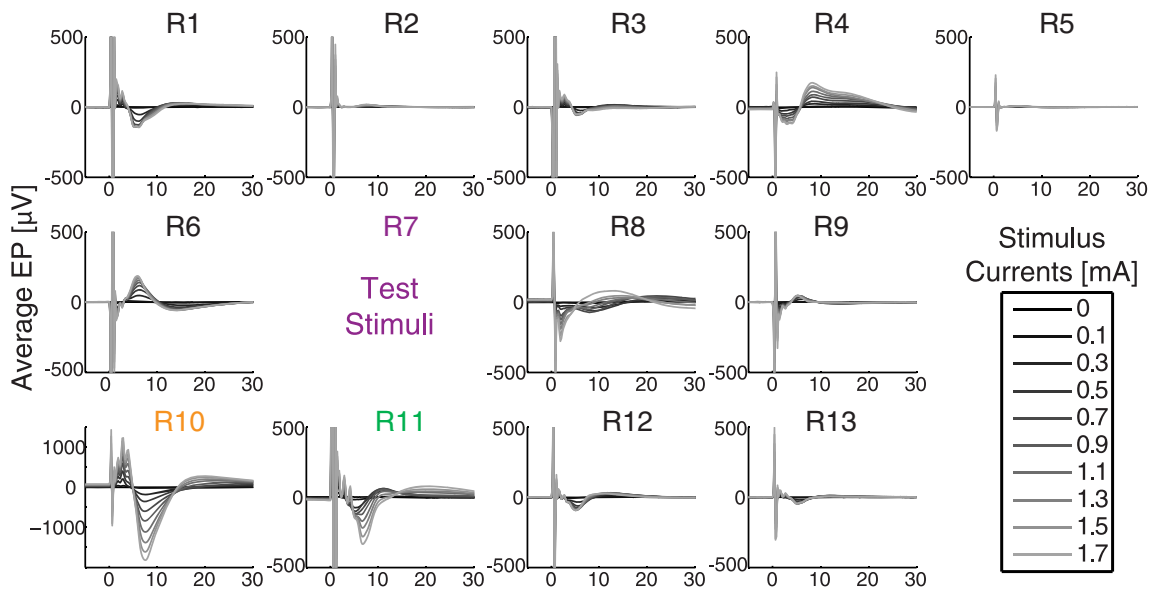

Time from stimulation [ms]

C

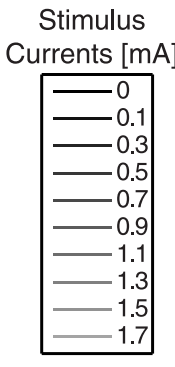

Currents $[\mathrm{mA}]$

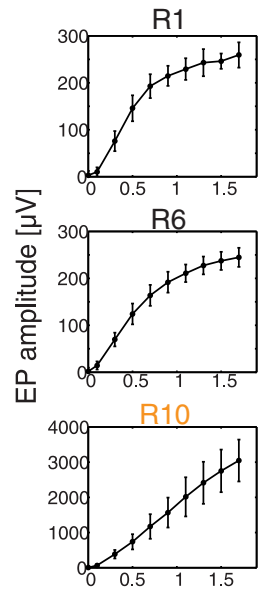

$\mathrm{R} 2$
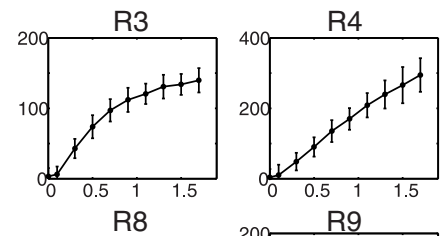

R7

Test

Stimuli

R11
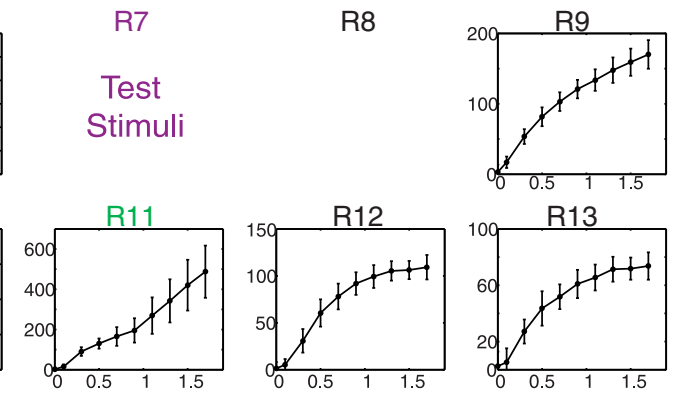

Stimulus Current $[\mathrm{mA}]$

R5

Figure 3. Cortical connectivity from stimulus-evoked potentials. $\boldsymbol{a}$, Example test stimulation $(1.1 \mathrm{~mA})$ applied to purple site and recorded at green site in monkey $Q$ (orange site is control, $C$, site used in Fig. 6). StTA shows average EP in black, individual trials $(n=97)$ are shown in gray, and the light-blue shadow is the $95 \%$ confidence interval. Black arrowheads denote the max peak and trough used to calculate EP amplitude. $\boldsymbol{b}$, Overlaid StTAs at each recording site for all current intensities of test stimuli delivered to R7.c, Stimulus-response curves for each recording site for stimulation at R7 (mean \pm SEM). Blank panels indicate no response as judged from $\boldsymbol{b}$.

Facility to insulate the solder joint and PtIr rod. The tips of each rod were then deinsulated by hand using a scalpel to an impedance of $22-160 \mathrm{k} \Omega$ (Monkey Q) and 4-60 k $\Omega$ (Monkey U). For each dual electrode, a 3 and a $5 \mathrm{~mm}$ PtIr rod were secured in a small piece of polytetrafluoroethylene (PTFE) tubing with silicon glue. The tips of the 3 and $5 \mathrm{~mm}$ rods were placed $\sim 0.5$ and $2-2.5 \mathrm{~mm}$ from the edge of the PTFE tube (Fig. $1 a$ ). In this way, the $5 \mathrm{~mm}$ rod penetrated to layer 5 of motor cortex containing corticofugal pyramidal cell somas, whereas the $3 \mathrm{~mm}$ rod rested on the surface of the brain. After each dual array was constructed, the back ends of the lead wires were soldered to connectors, one per hemisphere.

\section{Recordings}

Implant surgery. All surgeries were performed under isoflurane anesthesia and aseptic conditions. An incision was made along the midline of the scalp and muscle and connective tissue were resected to expose enough skull to place a 2.5 -inch-diameter titanium casing. Four to eight screws were placed in the skull around the edge of the exposure. At least four of the skull screws were T-screws used as grounds for the electrode implant. Holes were drilled with a $1.1 \mathrm{~mm}$ bit in a $3 \times 5$ grid with 2-3 mm center-to-center spacing using stereotaxic coordinates (Fig. 1b). After all of the holes were drilled, one dual electrode was placed with forceps into each hole until resistance was felt between the longer rod and the dura. The dual electrode was then pushed through the dura and into the brain until a second resistance was felt between the shorter rod and the dura. We use the term "surface" instead of epidural or subdural to describe the location of the shorter rod because it was impossible to know whether the dura was punctured by the shorter rod. Once all of the dual electrodes for one hemisphere were implanted, a thin coat of dental acrylic (methyl methacrylate) was used to seal the holes and hold them in place. This process was repeated for the other hemisphere. The casing was then placed over the implant and secured to the skull screws with acrylic. The connectors for the dual electrodes were cemented to the skull within the casing. Animals received postoperative courses of analgesics and antibiotics.

\section{Behavior}

Monkey U performed a center-out target acquisition wrist task in a sound-attenuating recording booth. Monkey U's right hand was restrained in a manipulandum measuring torque about the wrist in the radial/ulnar (RU) and flexion/extension (FE) axes. The torque produced in the FE-RU plane was displayed as the $x-y$ coordinates of a tracking cursor on a video monitor in front of the animal. A trial began when a center target appeared on the monitor, representing the "zero force" or neutral position of the cursor. Monkey U was required to hold the cursor in the center target for $2 \mathrm{~s}$ before a peripheral target at one of eight cardinal positions was presented. Monkey $U$ then moved the cursor by exerting isometric force on the manipulandum to the intended target and held it there for $2 \mathrm{~s}$ before returning to a second center target. The center-outcenter sequence was considered one trial. Applesauce reward was dispensed on a variable 1:2 ratio for every peripheral target presented and at the end of each trial. Monkey Q merely sat quietly in the recording booth.

General acquisition. Local field potentials (LFPs) were recorded using amplifiers from Guger Technologies in Monkey Q $(4800 \mathrm{~Hz}$ sampling rate) and the Grapevine Neural Interface System from Ripple in Monkey $\mathrm{U}(30,000 \mathrm{~Hz}$ downsampled to $5000 \mathrm{~Hz}$ post hoc). Single-ended recordings from the intracortical and surface electrodes of one hemisphere, referenced to a skull screw, were made simultaneously on up to 26 channels ( 13 dual electrodes) in Monkey Q and up to 30 channels in Monkey $\mathrm{U}$ (15 dual electrodes). Post hoc recordings were rereferenced as a bipolar signal for each dual electrode (intracortical - surface) to acquire a more 
localized recording and high-pass filtered over $10 \mathrm{~Hz}$. Wrist torques in the RU and FE axes were sampled for Monkey $\mathrm{U}$ by the Grapevine system at $1000 \mathrm{~Hz}$.

Estimate of electrode location relative to the central sulcus. The stereotaxic coordinates at which the electrodes were implanted were determined using an atlas to target the hand area of motor cortex. Because the atlas is only an approximate guide, coordinates were sometimes amended in surgery such that the middle of the grid was $\approx 18 \mathrm{~mm}$ lateral to bregma, which is the approximate location of hand motor area (Fetz and Cheney, 1980). To determine the location of each dual electrode more accurately relative to primary motor (M1) or somatosensory (S1) cortex, monopolar responses to stimulation of the contralateral median nerve were recorded with the monkey under ketamine sedation. The waveshape of the evoked potentials in stimulus-triggered averages (StTAs) of single-ended, surface recordings indicated the position of the recoding site relative to the central sulcus (McCarthy et al., 1991). Evoked potentials with a positive phase followed by a negative phase were generated in precentral cortex, whereas a negative phase followed by a positive phase occurred in postcentral cortex. Based on these recordings, a putative position of the central sulcus was drawn onto the grid (Fig. 1b).

\section{Stimulation}

Assessment of corticocortical connectivity. To identify connectivity between recording sites, monkeys were seated in a primate chair in a recording booth while electrical stimulation was delivered to each dual electrode. Stimulation was biphasic, with the negative phase leading on the intracortical wire and the positive phase leading on the surface wire of the dual electrode. Stimuli were delivered in a series of increasing current intensities, termed stimulus ramps, ranging from 0 to $2.25 \mathrm{~mA}$ with $7-10$ increments in the ramp and interstimulus intervals of 300-500 ms; each ramp was repeated continuously 100 times (Fig. 2b). Stimulus-evoked potentials (EPs) were measured at all ipsilateral sites in response to stimulation at one site during testing. A stimulus-response curve was produced for each site (Fig. 3c), building a map of connectivity across the grid. Single pulse stimulation of some sites elicited wrist and hand movement. For paired-stimulation conditioning experiments, ramp stimulation was performed in the booth for the two sites involved in conditioning (sites A and B) as well as a third control site (site C) not involved in conditioning. The order of test stimulation was randomized among the three sites. Testing stimulation was delivered at various time points relative to conditioning, as shown in Figure $2 a$. However, not all time points were recorded for each session to minimize the amount of time that an animal was handled because a complete session spanned several days.

Conditioning. Three reciprocally connected sites were identified via EPs and selected for paired-stimulation conditioning. The "presynaptic" site was termed A, the "postsynaptic site" B, and the third site, C, was used as a control. For Monkey Q, conditioning stimulation was performed with the Neurochip2 (Zanos et al., 2011) in the home cage to assess the efficacy of STDP while the animal was freely behaving, similar to previous monkey and rodent studies (Jackson et al., 2006; Rebesco et al., 2010; Rebesco and Miller, 2011; Lucas and Fetz, 2013; Nishimura et al., 2013). Stimulation pulses were bipolar and biphasic as described during testing. Conditioning stimulation was delivered first to site $\mathrm{A}$ and then to site $\mathrm{B}$ with a fixed delay (Fig. 2c). For Monkey U, conditioning stimulation was performed in the recording booth, using rack-mounted equipment. Similar to Monkey Q, site A received stimulation first, followed by site B.
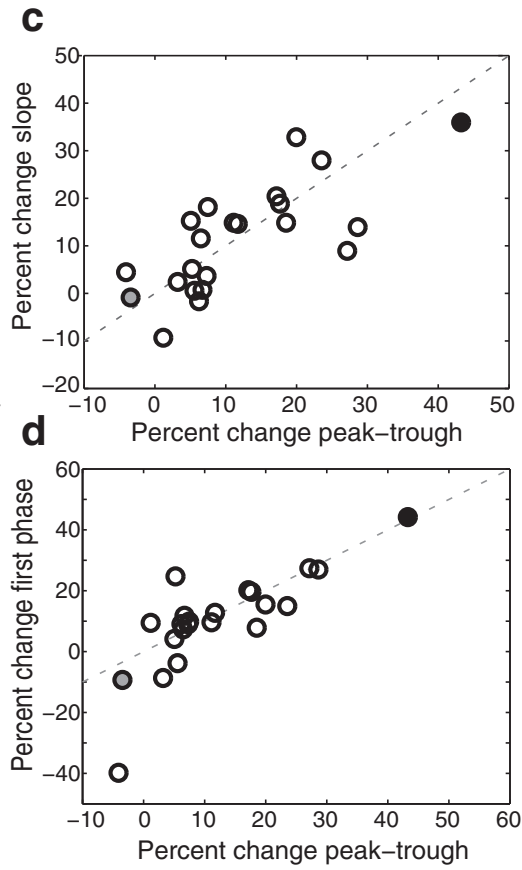

Figure 4. Characterization and comparison of EP measures. $\boldsymbol{a}$, Histogram of latency to first phase of EP for all responses in both monkeys. Dotted line denotes minimum cutoff due to electrical artifact $(1.5 \mathrm{~ms})$ and arrowhead denotes median $(2.7 \mathrm{~ms})$. $\boldsymbol{b}$, Histogram of number of phases in each EP. Arrowhead denotes median (3.0). $\boldsymbol{c}$, Percentage change in $A \rightarrow B E P$ amplitude after phase. Dotted line denotes unity; filled black point shows result from a successful conditioning session (Fig. 8); filled gray point shows result from an unsuccessful conditioning session (Fig. 14).

The paired-stimulation protocol during conditioning was as follows: three pulses at $330 \mathrm{~Hz}$ were delivered to site $\mathrm{A}$ and to site $\mathrm{B}$ separated by a specified delay $(\Delta t)$ as measured between the first pulse in each train. This sequence was repeated at $2 \mathrm{~Hz}$ for $1-3 \mathrm{~h}$ (Fig. $2 c$ ). Other stimulation protocols were also tested including $2 \mathrm{~Hz}$ single pulses, trains of 5 pulses at $1 \mathrm{kHz}$, trains of 10 pulses at $500 \mathrm{~Hz}$, as well as longer duration conditioning sessions from 24 up to $72 \mathrm{~h}$. We saw no consistent differences under these protocols and thus used three pulses at $330 \mathrm{~Hz}$ for the remainder of the study. A previous study also found that stimulus trains promote better plasticity in vivo than single pulses (Rebesco and Miller, 2011). The stimulus current selected for conditioning was one-third of the current in the middle of the dynamic range of the stimulus-response curve during testing (because condition stimulation was a three-pulse train instead of a single pulse during testing). The selected amplitudes were sufficient to activate neurons at site $A$ and $B$ without adverse effects such as disturbing the animal, causing cortical depression (due to prolonged stimulation with high currents; McCreery et al., 1986), or causing seizures. The current was further adjusted to be just at or below motor threshold if the one-third criterion evoked clear movements.

Analysis

EPs. The peak-trough amplitude of EPs was measured and analyzed to document the strength of corticocortical connectivity. Trials were aligned on stimulus onset and grouped for a given current intensity. All trials were inspected by eye and those with movement artifacts were removed. From the remaining trials, StTAs were calculated from $50 \mathrm{~ms}$ before to $30 \mathrm{~ms}$ after the time of stimulation for each current intensity (Fig. 3a). To separate a physiological response from electrical artifact, a biphasic stimulation pulse was delivered directly into the Guger Technologies amplifiers to visualize the artifact. The artifact returned to baseline by $1 \mathrm{~ms}$ after stimulation, so any waveform within the first millisecond was ignored. The Grapevine system has built-in artifact suppression, which grounded the recording channels for $1 \mathrm{~ms}$ at the time of stimulation. Each stimulus-aligned trial was also examined by eye for 
Table 1. Conditioning sessions for Monkey Q

\begin{tabular}{|c|c|c|c|c|c|c|c|c|c|c|c|c|c|}
\hline Experiment & Session & A site & B site & C site & Pulses & $\begin{array}{l}\text { Current } \\
\text { (uA) }\end{array}$ & $\begin{array}{l}\text { Frequency } \\
(\mathrm{Hz})\end{array}$ & $\begin{array}{l}\text { Delay } \\
\text { (ms) }\end{array}$ & $\begin{array}{l}\text { Duration } \\
\text { (h) }\end{array}$ & $\begin{array}{l}\mathrm{A} \rightarrow \mathrm{B} \\
\text { (\% change) }\end{array}$ & $\begin{array}{l}\mathrm{B} \rightarrow \mathrm{A} \\
\text { (\% change) }\end{array}$ & $\begin{array}{l}\mathrm{A} \rightarrow \mathrm{C} \\
\text { (\% change) }\end{array}$ & $\begin{array}{l}C \rightarrow B \\
\text { (\% change) }\end{array}$ \\
\hline \multirow[t]{9}{*}{1} & 20140917 & \multirow[t]{9}{*}{ R7 } & \multirow[t]{9}{*}{ R11 } & \multirow[t]{9}{*}{$\mathrm{R} 10$} & \multirow[t]{9}{*}{$3 @ 330 \mathrm{~Hz}$} & \multirow[t]{8}{*}{330} & \multirow[t]{8}{*}{2} & 20 & \multirow[t]{9}{*}{3} & $87.41^{*}$ & 3.13 & 3.91 & 1.70 \\
\hline & 20141006 & & & & & & & 10 & & $-42.13^{*}$ & $-62.80^{*}$ & 2.43 & -1.13 \\
\hline & 20150119 & & & & & & & 20 & & $34.70^{*}$ & $-53.50^{*}$ & -25.0 & -8.08 \\
\hline & 20150316 & & & & & & & 200 & & -20.96 & 2.21 & -0.21 & 6.88 \\
\hline & 20150330 & & & & & & & 50 & & $-45.07^{*}$ & -1.42 & 15.24 & $23.53^{*}$ \\
\hline & 20150406 & & & & & & & 100 & & -10.39 & -1.45 & 22.6 & 23.0 \\
\hline & 20150422 & & & & & & & 30 & & -17.77 & 6.94 & $31.43^{*}$ & $15.35^{*}$ \\
\hline & 20150427 & & & & & & & 10 & & $52.96^{*}$ & -10.30 & 19.74 & $31.48^{*}$ \\
\hline & 20150720 & & & & & - & - & - & & -7.92 & $29.42^{*}$ & 9.10 & -0.032 \\
\hline \multirow[t]{2}{*}{2} & 20150216 & \multirow[t]{2}{*}{ L7 } & \multirow[t]{2}{*}{ L11 } & \multirow[t]{2}{*}{ L10 } & \multirow[t]{2}{*}{$3 @ 300 \mathrm{~Hz}$} & \multirow[t]{2}{*}{330} & \multirow[t]{2}{*}{2} & 20 & \multirow[t]{2}{*}{3} & $34.18^{*}$ & $-34.70^{*}$ & $-44.49^{*}$ & $-24.76^{*}$ \\
\hline & 20150608 & & & & & & & 20 & & -1.93 & $52.24^{*}$ & -16.66 & 0.35 \\
\hline 6 & 20150706 & L8 & L4 & L6 & $3 @ 300 \mathrm{~Hz}$ & 330 & 2 & 20 & 3 & -17.51 & 8.86 & 12.77 & -6.03 \\
\hline
\end{tabular}

Experiments are grouped by sites and sessions and are listed chronologically. Percentage change in EP amplitude was measured immediately after paired stimulation ended. In session 20150403 , two conditioning blocks were performed with a $3 \mathrm{~h}$ gap in between. The first entry is the result of the first conditioning block and $20150403+$ is the second conditioning block (see Results). ${ }^{*} p<0.05$ based on control distributions from Figure 13 .

evidence of a clean separation between the stimulus artifact and the physiological response. Trials in which there was possible contamination by the artifact were discarded. The amplitude of the average EP was quantified by subtracting the largest trough from the largest peak in a window 1.5 to $30 \mathrm{~ms}$ after stimulation for all current intensities (Fig. $3 a$, arrowheads). Latency of the EP features could vary slightly, so care was taken to ensure that the same peak and trough for a given EP were measured across time points. This metric was chosen as a simple, unbiased way to capture the complicated, often multiphasic, shape of the EP. Conditioning effects were measured as the percentage change in average EP amplitude from before to after conditioning, similar to other studies measuring changes in field potential recordings (Hess et al., 1996). For some conditioning experiments, testing was performed at regular intervals before and/or after the conditioning window to assess diurnal trends and wash-out of the effect. Preconditioning EP amplitudes were then quantified as the mean of all testing sessions before the start of conditioning. A subset of EPs were also quantified in Monkey $U$ by the slope and amplitude of the first phase of the response because this was the response component most likely to be due to a monosynaptic connection between sites A and B. Only EPs from Monkey U were analyzed in this way because the artifact suppression feature of the Ripple amplifiers, and their ability to record up to $30 \mathrm{kHz}$, allowed consistent identification of the rising edge of the first phase of the response.

Statistics. All EP amplitudes were quantified as a mean \pm SEM and $p$-values were calculated with a two-sample $z$ test, except where noted. ANOVAs were used to determine the significance of differences between group means, followed by post hoc Kolmogorov-Smirnov (K-S) or $t$ tests.

Results from the $z$ test indicated that many connections outside of the $\mathrm{A} \rightarrow \mathrm{B}$ pair changed strength after conditioning stimulation. To quantify this network-wide change and to determine whether the $\mathrm{A} \rightarrow \mathrm{B}$ connection showed greater effects, we created cumulative density curves for the $\mathrm{A}, \mathrm{B}$, and $\mathrm{C}$ sites. Cumulative distributions have been used in in vitro STDP studies to assess effects between different experimental conditions (Lu et al., 2007). A control distribution for the outputs of site $A\left(A_{\text {cont }}\right)$ was compiled from the percentage change after conditioning of the evoked responses at all sites except $\mathrm{B}$ and $\mathrm{C}$. Similar distributions were calculated for sites $\mathrm{B}\left(\mathrm{B}_{\text {cont }}\right)$ and $\mathrm{C}\left(\mathrm{C}_{\text {cont }}\right)$. One-sample $\mathrm{K}-\mathrm{S}$ tests were used to determine whether the mean EP changes in the control distributions were significantly different from zero. The $p$-values for these tests are shown in the diagonal elements of matrices (termed "significance matrices") at the bottom of Figures 10 and 13 .

The $\mathrm{A}_{\text {cont }}, \mathrm{B}_{\text {cont }}$, and $\mathrm{C}_{\text {cont }}$ distributions were compared with the distributions of EP amplitude changes between two of the A, B, and C sites (e.g., distribution of the $\mathrm{A} \rightarrow \mathrm{B}$ effects). The $\mathrm{K}-\mathrm{S}$ test was used to assess the significance of differences between a control and a site-pair-specific distribution. The $p$-values for these tests are shown in the off-diagonal elements of the significance matrices in Figures 10 and 13. For instance, in Figure 10, the upper right quadrant is the $p$-value of a $\mathrm{K}-\mathrm{S}$ test between the $\mathrm{A} \rightarrow \mathrm{B}$ distribution and the $\mathrm{A}_{\text {cont }}$ distribution. Two-sided significance of $p<0.05$ for EP changes was based on the lower 2.5th and upper 97.5th percentile of control distributions.

\section{Results}

Paired-stimulation conditioning was delivered between pairs of sites in sensorimotor cortex of awake, behaving monkeys to induce STDP. Results were obtained from two M. nemestrina monkeys for 15 different pairs of functionally connected sites, two of which showed delay-dependent effects consistent with STDP as measured by the peak-trough amplitude of EPs.

\section{Selection of conditioning pairs from EPs}

We used EPs to identify and quantify the strength of connections between cortical sites. Changes in EP amplitude due to pairedstimulation conditioning were interpreted as a change of connection strength. Stimulus ramps (see Materials and Methods) were delivered at each site individually while responses at all other sites on the electrode grid were recorded. StTAs of EPs revealed high connectivity across the grid, as indicated by short-latency, multiphase potentials. Figure $3 a$ shows an example EP at site R11 in response to stimulation at site $\mathrm{R} 7$ at $1.1 \mathrm{~mA}$. The first phase usually began within $2 \mathrm{~ms}$, followed by broad, longer-latency phases that usually returned to baseline by $\sim 50 \mathrm{~ms}$ after stimulation. After distinguishing the physiological response from the stimulation artifact (see Materials and Methods), a peak-trough measure quantified the amplitude of EPs between 1.5-30 ms after stimulation $(\mathrm{EP}$ amp $=$ largest peak - largest trough; Fig. $3 a$, arrowheads). The stimulus ramps used a range of current amplitudes to describe the stimulus-response relationship (Fig. 3b,c). We chose a current range and step resolution that identified a threshold, linear range, and saturation point for a wide range of connected sites (see Materials and Methods). As can be seen in Figure $3 b$, stimulation at a single site elicited responses at many other sites, though not all. EP latency, shape, number of phases, 


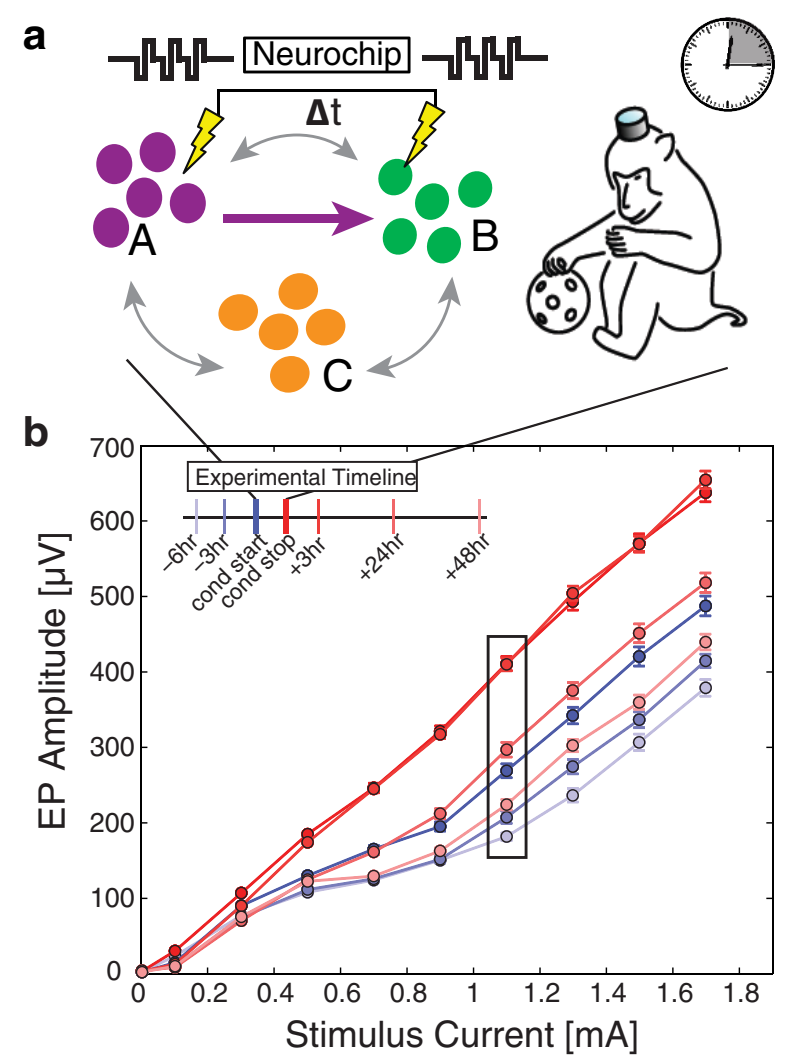

C
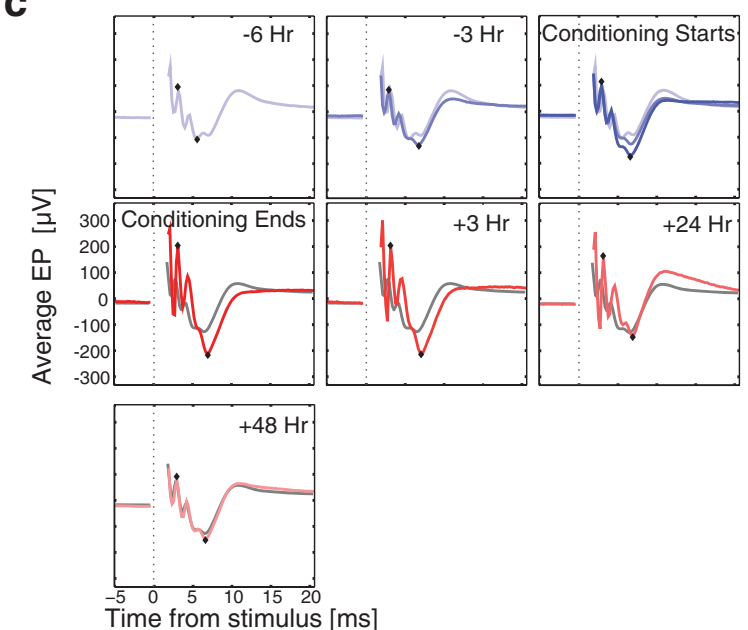

d

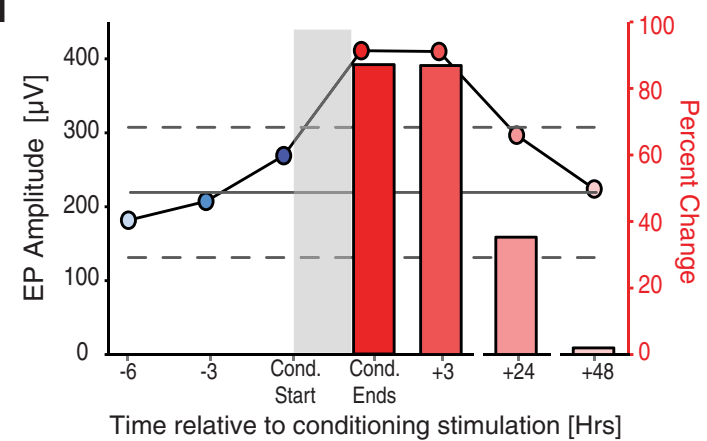

Figure 5. Three-hour paired-stimulation conditioning session at 20 ms delay. $\boldsymbol{a}$, Schematic of conditioning between site $A$ and site $B$ using the Neurochip while Monkey $Q$ was in the home cage for $3 \mathrm{~h}$. Sites A, B, and C were the same as in Figure $3 a$. $\boldsymbol{b}$, Preconditioning (blue) and postconditioning (red) stimulus-response curves at time points relative to conditioning as denoted by timeline. $\boldsymbol{c}$, Average EP from $A \rightarrow B$ at current denoted by black box in $\boldsymbol{b}$; diamonds

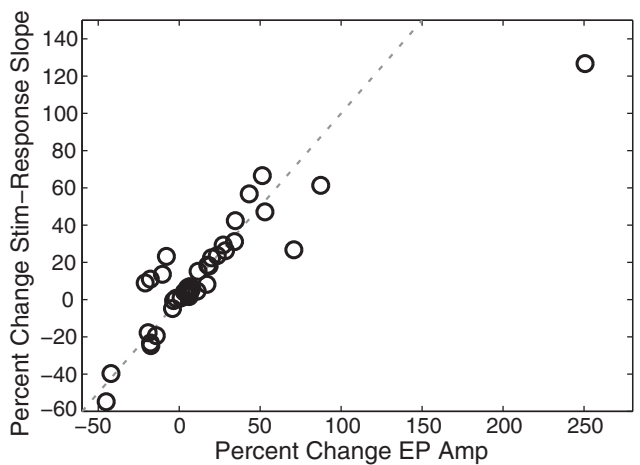

Figure 6. Comparison of conditioning effects with two different EP measures. Shown is the percentage change in $A \rightarrow B E P$ amplitude after conditioning compared with percentage change in $A \rightarrow B$ slope of stimulus-response curve after conditioning for all conditioning sessions in both monkeys. The stimulus-response curve was fit with a linear function to good approximation $\left(r^{2} \geq 0.82\right)$.

and amplitude varied greatly from site to site, likely due to multiple factors, both technical (e.g., the relative positioning of the electrodes) and physiological (e.g., underlying monosynaptic and polysynaptic connectivity between sites). The polarity of EPs varied from site to site as well (Fig. 3b), likely due to the location of the electrodes with respect to the current dipoles both in cortical depth and across the central sulcus (Buzsáki et al., 2012). The median latency to the first EP phase for all responses in both monkeys was $2.7 \mathrm{~ms}$, although this varied over a large range (1.6$12.7 \mathrm{~ms}, n=187 \mathrm{EPs}$; Fig. $4 a$ ). The number of phases in a given EP ranged from one to eight with a median of three (Fig. $4 b$ ). Because EPs were highly variable from site to site, coupled with the conflation of technical and physiological mechanisms underlying EP shape, we hesitate to infer too much about the precise connectivity between sites solely based on EP characteristics (e.g., which EP phases correspond to monosynaptic vs polysynaptic connections). Because both could be affected by the conditioning paradigm, we used the peak-trough amplitude to quantify the strength of connections between sites and how this changed with paired stimulation. Furthermore, conditioning effects on the slope and amplitude of the first response phase, which may be more susceptible to STDP effects (Diesmann et al., 1999), were highly correlated with the effects on the peak-trough amplitude (Fig. $4 c, d$, peak-trough vs slope $p=0.66$, peak-trough vs first phase $p=0.38$, paired $t$ test).

To assess the specificity of changes in connectivity due to the paired stimulation, we compared the effects of conditioning on the $\mathrm{A} \rightarrow \mathrm{B}$ connection with those from $\mathrm{A} \rightarrow \mathrm{C}$ and $\mathrm{C} \rightarrow \mathrm{B}$. Changes in $\mathrm{EP}$ amplitudes from $\mathrm{A} \rightarrow \mathrm{C}$ reveal nonspecific, presynaptic effects at site $A$, whereas changes in the $C \rightarrow B$ connection reveal nonspecific, postsynaptic effects at the B site. It is important to note that, although we selected a $\mathrm{C}$ site that was recurrently connected to both $\mathrm{A}$ and $\mathrm{B}$, many other sites on the grid showed EPs from A and/or B (Fig. $3 b$ ) and could also be used to detect changes in global excitability due to the stimulation.

denote peak and trough used to measure amplitude in $\boldsymbol{b}$. Blue EPs in top three panels are averaged into a composite baseline (gray trace) in subsequent panels. $\boldsymbol{d}$, Circles and left axis showing EP amplitude at times relative to conditioning at current denoted by black box in $\mathbf{b}$. Horizontal gray line is mean of three preconditioning points; dashed line is $95 \%$ confidence interval. Bars and right axis show percentage increase in EP amplitude above composite baseline. Conditioning occurred during gray bar. Delay between stimuli was $20 \mathrm{~ms}$. 


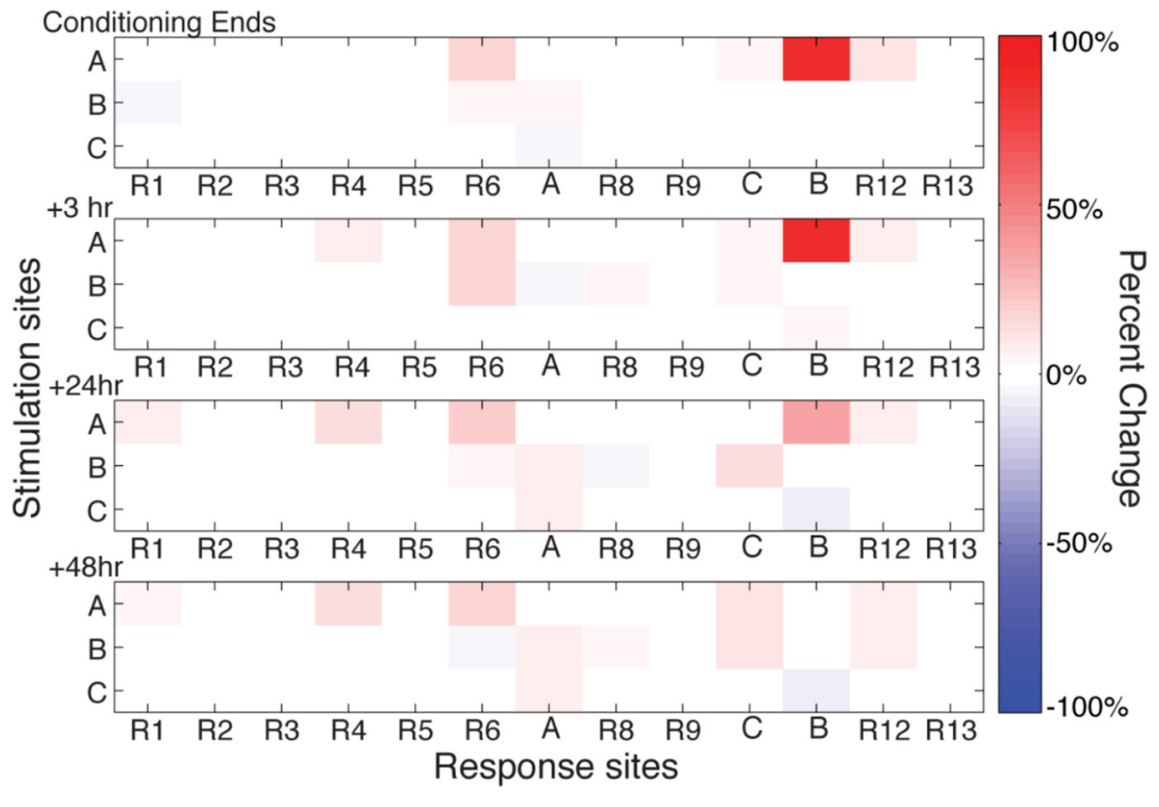

Figure 7. Network-wide effects of conditioning. Percentage change in EPs (color map shown to right) from A, B, and C to all other sites where there was a response. Panels show time points after conditioning (top to bottom). Data are from the same conditioning session shown in Figure 5.

\section{Paired stimulation increases EP amplitude}

A representative paired-stimulation conditioning session (Table 1 , session 20140917) with a 20 ms delay between stimuli is shown in Figure 5. Three hours of conditioning stimulation (3-pulse trains at $330 \mathrm{~Hz}$ every $500 \mathrm{~ms}, 330 \mu \mathrm{A}$ ) were delivered at sites R7 (A site) and R11 (B site) (Fig. 3a) while Monkey Q was in the home cage. Athough stimulus ramps were used to probe the connectivity between sites (Fig. 3), a majority of the analysis focused on responses to $1.1 \mathrm{~mA}$ (outlined by the black box in Fig. 5b), which was close to the cumulative current used during conditioning (i.e., 3 pulses of $330 \mu \mathrm{A}$ ) and was in the middle of the stimulus range.

In addition to testing connection strengths immediately before and after conditioning, we tested at additional times relative to the $3 \mathrm{~h}$ conditioning window (Fig. $5 b-d$ ). Although EP amplitudes within a given testing period showed little variance (e.g., $0.34 \mathrm{CV}$; Fig. $3 a$ ), there was more variability over the course of $3 \mathrm{~h}$ before conditioning, consistent with previous observations (Richardson and Fetz, 2012). To account for this variability, we used the mean of all preconditioning EPs as a baseline (Fig. $5 c, d$ ). Immediately after conditioning, the EP amplitude from $\mathrm{A} \rightarrow \mathrm{B}$ increased $87.4 \%$ (from $219.3 \pm 83.6 \mu \mathrm{V}$ to $411.0 \pm 9.0 \mu \mathrm{V}$, mean $\pm \mathrm{SD}, n=295 ; p<0.001)$. This increase in connection strength from $\mathrm{A} \rightarrow \mathrm{B}$ was maintained $3 \mathrm{~h}$ later $(410.0 \pm 9.0 \mu \mathrm{V}$, $+86.9 \%, p<0.001)$. Twenty-four hours after conditioning ceased, the amplitude fell back within the SD of the preconditioning baseline, but still remained significantly elevated (297.0 \pm $10.0 \mu \mathrm{V}, p<0.001$ ) and, by $48 \mathrm{~h}$ after conditioning, had fully returned to baseline $(224.0 \pm 7.0 \mu \mathrm{V}, p=0.56$; Fig. $5 d$. $)$. Changes in the slope of the stimulus-response curve, another measure of connection strength (Hess et al., 1996), follow those of the EP amplitude at the chosen current (Fig. 6, $p=0.54$, paired $t$ test, $n=41$ sessions), suggesting that these results are consistent over the range of stimulus currents tested.

Because potentiation of the $\mathrm{A} \rightarrow \mathrm{B}$ connection was maintained for at least $3 \mathrm{~h}$ in the absence of continued paired stimulation, we ran a second conditioning block to determine whether the $\mathrm{A} \rightarrow \mathrm{B}$ connection could be potentiated further. We tested this during one session (Table 1 , session 20150403†) in which a $3 \mathrm{~h}$ block of paired stimulation was followed by $3 \mathrm{~h}$ of no stimulation and then an additional $3 \mathrm{~h}$ of paired stimulation. During the first conditioning block, the $\mathrm{A} \rightarrow \mathrm{B}$ EP increased from $123.1 \pm 25.0 \mu \mathrm{V}$ to $431.6 \pm$ $56.2 \mu \mathrm{V}(+250.7 \%, p<0.05)$. After $3 \mathrm{~h}$ of no stimulation the $\mathrm{A} \rightarrow \mathrm{B}$ amplitude fell to $261.6 \pm 44.6 \mu \mathrm{V}$, which was still significantly larger than the preconditioning amplitude $(+112.5 \%, p<0.05)$. However, after a second $3 \mathrm{~h}$ block of paired stimulation, the $\mathrm{A} \rightarrow \mathrm{B}$ EP amplitude did not increase further and was maintained at $256.6 \pm 36.0 \mu \mathrm{V}(-3.45 \%$ compared with the previous testing period, $p>0.05$ ).

Although paired stimulation was delivered between specific $A \rightarrow B$ sites, the widespread connections across the electrode grid allowed us to see effects throughout the network. A summary of the postconditioning responses at all sites to stimulation at A, B, or C during the session illustrated in Figure 5 is shown in Figure 7 (white tiles indicate no EP recorded). Many connections showed small increases or decreases in response to paired stimulation, but an ANOVA revealed a significant difference between the mean $A \rightarrow B, A \rightarrow C$ $(+3.9 \%)$, and $\mathrm{C} \rightarrow \mathrm{B}(+1.7 \%)$ responses immediately after conditioning $(p<0.001)$. Post hoc analyses demonstrated that the change in EP amplitude from $\mathrm{A} \rightarrow \mathrm{B}$ was significantly larger than that between $\mathrm{A} \rightarrow \mathrm{C}(p<0.001,2$-sample $t$ test $)$ and $\mathrm{C} \rightarrow \mathrm{B}(p<$ $0.001)$. Two other sites showed a significant amplitude increase in EPs elicited from site A immediately after conditioning (R6: $+17.6 \%, p<0.001$; and R12: + 11.7\%, $p<0.001$; Fig. 7). Stimulation protocols producing changes in cortical connectivity that are unrelated to the stimulus pairing have been reported previously (Rebesco and Miller, 2011). These effects could result from global changes in cortical excitability due to the repetitive stimulation, which will be discussed in further detail below. The bottom three panels of Figure 7 show network-wide changes at testing periods $+3,+24$, and $+48 \mathrm{~h}$ after conditioning.

A second example conditioning session in Monkey U (Table 2, session 20150603) is shown in Figure 8. Because Monkey U was engaged in a behavioral task, testing and conditioning periods were obtained in the booth during one session. Conditioning stimulation was delivered for $1 \mathrm{~h}$ at a delay of $20 \mathrm{~ms}$ between site L3 (A site) and L7 (B site), with site L2 serving as the control (C site) (Fig. $8 a$ ). Figure $8 b$ shows the average EP for $400 \mu \mathrm{A}$ stimuli before and after conditioning, with the peak and trough denoted by the black diamonds and the corresponding stimulus-response relationship shown in in Figure $8 c$. The insets show similar results obtained using the rising slope of the first component of the evoked potential as the measured variable, as has been used in in vitro paired-stimulation plasticity experiments (Froemke and Dan, 2002). Both metrics show a similar effect of conditioning (Fig. 4c, black filled circle). Again, we saw a large increase in EP amplitude from $\mathrm{A} \rightarrow \mathrm{B}(+43.3 \%, p<0.001)$ and variable increases and decreases among other connections. An ANOVA comparing mean responses among $\mathrm{A} \rightarrow \mathrm{B}, \mathrm{A} \rightarrow \mathrm{C}(-16 \%)$, and $\mathrm{C} \rightarrow \mathrm{B}(-2.1 \%)$ was significant $(p<0.001)$ and post hoc $t$ tests 
Table 2. Conditioning sessions for Monkey U

\begin{tabular}{|c|c|c|c|c|c|c|c|c|c|c|c|c|c|}
\hline Experiment & Session & A site & B site & C site & Pulses & $\begin{array}{l}\text { Current } \\
\text { (uA) }\end{array}$ & $\begin{array}{l}\text { Frequency } \\
(\mathrm{Hz})\end{array}$ & $\begin{array}{l}\text { Delay } \\
\text { (ms) }\end{array}$ & $\begin{array}{l}\text { Duration } \\
\text { (h) }\end{array}$ & $\begin{array}{l}A \rightarrow B \\
\text { (\% change) }\end{array}$ & $\begin{array}{l}\mathrm{B} \rightarrow \mathrm{A} \\
\text { (\% change) }\end{array}$ & $\begin{array}{l}A \rightarrow C \\
\text { (\% change) }\end{array}$ & $\begin{array}{l}C \rightarrow B \\
\text { (\% change) }\end{array}$ \\
\hline \multirow[t]{10}{*}{1} & 20150528 & \multirow[t]{10}{*}{ L3 } & \multirow[t]{10}{*}{ L7 } & \multirow[t]{10}{*}{ L2 } & \multirow[t]{8}{*}{$3 @ 330 \mathrm{~Hz}$} & \multirow[t]{2}{*}{80} & \multirow[t]{2}{*}{2} & 20 & \multirow[t]{10}{*}{1} & $51.37^{*}$ & 8.41 & -12.41 & -0.97 \\
\hline & 20150529 & & & & & & & B only stim & & 17.65 & $157.22^{*}$ & 2.54 & -3.18 \\
\hline & 20150604 & & & & & \multirow[t]{6}{*}{-} & \multirow[t]{6}{*}{-} & - & & 19.96 & 0.54 & 0.29 & 2.74 \\
\hline & 20150608 & & & & & & & 10 & & $27.15^{*}$ & 5.41 & -0.47 & 0.68 \\
\hline & 20150609 & & & & & & & 30 & & 23.53 & 4.07 & 4.80 & 1.17 \\
\hline & 20150806 & & & & & & & 20 & & $28.64^{*}$ & 5.19 & 12.24 & 6.04 \\
\hline & 20150807 & & & & & & & 20 & & 6.70 & -1.14 & 8.30 & 0.96 \\
\hline & 20150928 & & & & & & & 20 & & 18.52 & 2.92 & $36.19^{*}$ & 4.27 \\
\hline & 20151001 & & & & 1 & 200 & & 6 & & 11.70 & 5.28 & 13.85 & -1.14 \\
\hline & 20160108 & & & & $3 @ 300 \mathrm{~Hz}$ & 80 & & 50 & & 11.11 & 17.25 & $29.10^{*}$ & 2.88 \\
\hline 2 & 20150707 & L13 & L12 & L9 & $3 @ 330 \mathrm{~Hz}$ & 100 & 2 & 20 & 1 & 5.23 & -1.07 & 0.23 & 4.33 \\
\hline 5 & 20150522 & L8 & L6 & L12 & $3 @ 330 \mathrm{~Hz}$ & 100 & 2 & 20 & 1 & 3.20 & 3.40 & 3.76 & -5.91 \\
\hline 6 & 20150527 & L10 & L15 & L14 & 3 @330 Hz & 120 & 2 & 20 & 1 & 17.16 & -7.31 & $27.45^{*}$ & $-40.74^{*}$ \\
\hline 7 & 20150702 & L8 & L9 & L3 & $3 @ 330 \mathrm{~Hz}$ & 120 & 2 & 20 & 1 & 7.29 & 4.14 & 6.64 & 1.11 \\
\hline 8 & 20150709 & L6 & L2 & L7 & $3 @ 330 \mathrm{~Hz}$ & 140 & 2 & 20 & 1 & -4.10 & 2.90 & 4.70 & 3.46 \\
\hline 9 & 20150710 & L12 & L8 & L13 & $3 @ 330 \mathrm{~Hz}$ & 140 & 2 & 20 & 1 & 1.16 & 4.82 & -1.78 & 2.64 \\
\hline
\end{tabular}

Experiments are grouped by sites and sessions and are listed chronologically. Percentage change in EP amplitude was measured immediately after paired stimulation ended. * $p<0.05$ based on control distributions from Figure 13 .

showed significant differences between the $\mathrm{A} \rightarrow \mathrm{B}$ and $\mathrm{A} \rightarrow \mathrm{C}$ and the $\mathrm{A} \rightarrow \mathrm{B}$ and $\mathrm{C} \rightarrow \mathrm{B}$ effects (both $p<0.001$ ). As in the previous example, conditioning led to significant changes of EP amplitude at several other sites across the electrode grid (Fig. $8 d$ ). In summary, these two conditioning sessions exhibited a significant increase in EP amplitude between the targeted site pair that was accompanied by weaker, nonspecific effects at both the presynaptic and postsynaptic sites.

\section{STDP and global changes result from paired stimulation}

STDP is characterized by a specific relationship between the change in synaptic strength and the delay between presynaptic and postsynaptic firing. Short delays, such as the $20 \mathrm{~ms}$ interstimulus interval highlighted in the above examples, are optimal for inducing potentiation in the $\mathrm{A} \rightarrow \mathrm{B}$ direction, whereas longer delays, typically $>50 \mathrm{~ms}$, lie outside of the potentiation window (Bi and Poo, 1998). We tested a range of delays $(6,10,20,30,50$, 100 , and $200 \mathrm{~ms}$; Tables 1, 2) for the R7 $\rightarrow$ R11 (Monkey Q) and $\mathrm{L} 3 \rightarrow$ L7 (Monkey U) site pairs to determine whether the pairedstimulation conditioning effects were consistent with an STDP rule. Figure 9 shows that, at delays $<50 \mathrm{~ms}$, the $\mathrm{A} \rightarrow \mathrm{B}$ connection is potentiated preferentially in accordance with a pre-before-post rule ( $\mathrm{Bi}$ and $\mathrm{Poo}, 2001)$. The reverse $\mathrm{B} \rightarrow \mathrm{A}$ connection serves as a proxy for post-before-pre conditioning, for which the STDP rule predicts synaptic depression (Bi and Poo, 1998). Although most $\mathrm{B} \rightarrow \mathrm{A}$ responses showed little change with conditioning, two cases in Monkey Q (Fig. 9, circles, lower left quadrant) showed the expected decrease.

To confirm that the STDP effects were robust, we repeated conditioning sessions with the optimal $20 \mathrm{~ms}$ interstimulus delay for these two site pairs. Conditioning produced significant increases in EP amplitude from $\mathrm{A} \rightarrow \mathrm{B}$ in many, although not all, sessions (Fig. 9, Tables 1,2). On average, the change in $A \rightarrow B$ EP amplitude for $20 \mathrm{~ms}$ delays at these two site pairs was significantly different from zero (median change $=+39 \%$; $p<0.01$, onesample sign test; the small number of data points were not nor- mally distributed). A Kruskal-Wallis test showed a significant difference $(p=0.002)$ between the $\mathrm{A} \rightarrow \mathrm{B}, \mathrm{A} \rightarrow \mathrm{C}$ (median $=$ $+6.1 \%)$, and $\mathrm{C} \rightarrow \mathrm{B}(+1.0 \%)$ distributions for all 20 -ms-delay sessions. Post hoc analyses indicated a significant difference between the $\mathrm{A} \rightarrow \mathrm{B}$ and $\mathrm{C} \rightarrow \mathrm{B}$ distributions $(p=0.007$, MannWhitney test) and between the $\mathrm{A} \rightarrow \mathrm{B}$ and $\mathrm{A} \rightarrow \mathrm{C}$ distributions $(p<0.001)$.

The effect of interstimulus interval on changes in the strength of the $\mathrm{A} \rightarrow \mathrm{B}$ connection strongly suggests a role for Hebbian STDP. However, as in the example conditioning sessions described above (Figs. 7, 8d), we observed EP changes outside of the target $\mathrm{A} \rightarrow \mathrm{B}$ connection that were not predicted by STDP. This result led us to question whether the $A \rightarrow B$ effects could be accounted for by global changes in excitability. To separate global from targeted, paired-stimulation effects on EP amplitude, we created a distribution of postconditioning effects for all outputs from site A (except B and C) and site B (except A and C) for the two site pairs (Q:R7 $\rightarrow$ R11, U:L3 $\rightarrow$ L7) across all delays $(n=23$ sessions; Fig. $10 a, c)$. These two distributions were termed $\mathrm{A}_{\text {cont }}$ and $\mathrm{B}_{\text {cont }}$ (see Materials and Methods) and are shown as histograms (Fig. 10a,c) and cumulative densities (Fig. 10b,d) in Figure 10. The $A_{\text {cont }}$ distribution showed a significant increase in EP amplitude compared with zero $(+4.0 \pm 1.0 \%, p<0.001$, 1 -sample $t$ test, upper left quadrant of significance matrix at the bottom right of Fig. 10), highlighting the nonspecific effects of repetitive stimulation.

Comparison of the cumulative density curve of the $\mathrm{A}_{\text {cont }}$ distribution to that compiled from just the $\mathrm{A} \rightarrow \mathrm{B}$ effects (Fig. 10b) shows a significant separation $(p<0.001, \mathrm{~K}-\mathrm{S}$ test, upper right quadrant of significance matrix; Fig. 10). Therefore, the increase in connectivity strength from A to B due to the targeted conditioning surpasses that of the global effects on the network. Furthermore, increases in $\mathrm{A} \rightarrow \mathrm{B}$ EPs at short delays (10-30 ms) drive this difference because $A \rightarrow B$ effects at long delays (50-200 ms) were not significantly different from $\mathrm{A}_{\text {cont }}(n=6$ sessions, $p=$ $0.175, \mathrm{~K}-\mathrm{S}$ test). Conversely, conditioning effects on the $\mathrm{B} \rightarrow \mathrm{A}$ 
a

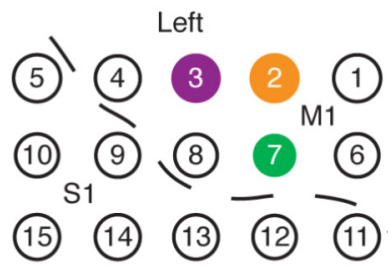

b

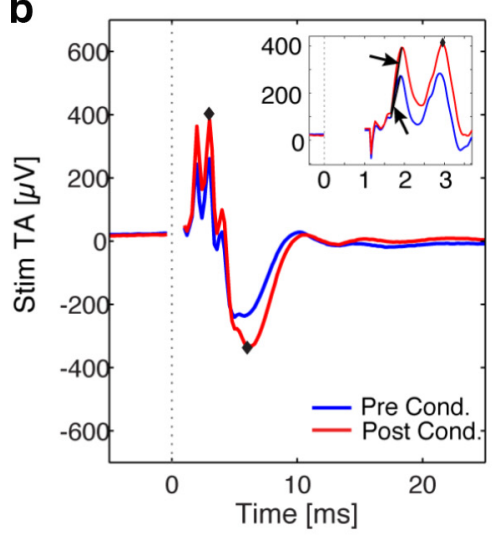

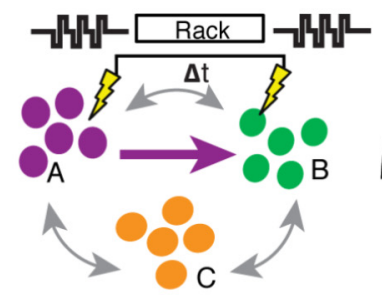

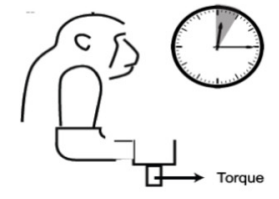

C

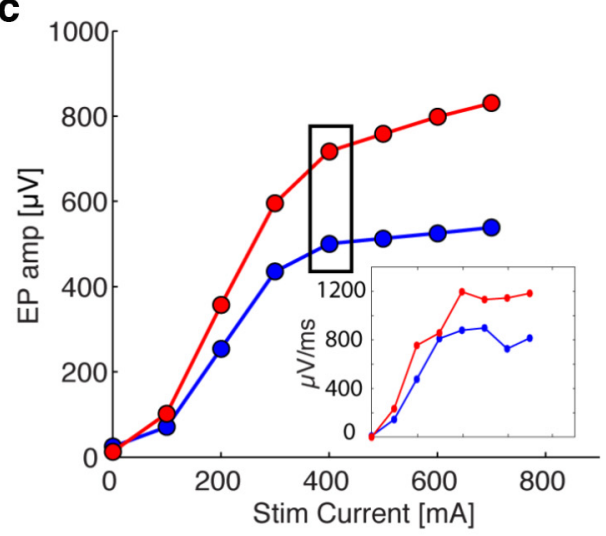

d

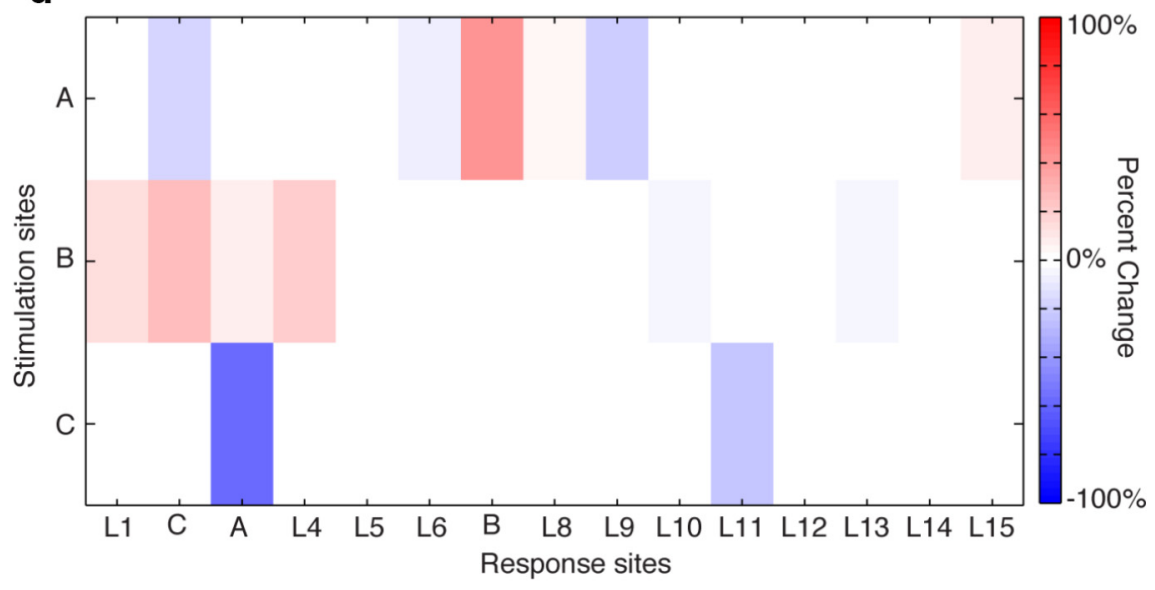

Figure 8. One-hour paired-stimulation conditioning session at $20 \mathrm{~ms}$ delay. $\boldsymbol{a}$, Schematic of conditioning session with $20 \mathrm{~ms}$ delay using rack-mounted equipment while Monkey $U$ was in the booth for $1 \mathrm{~h}$. Left,Cortical positions of A (purple), B (green), and $C$ (orange) sites relative to the central sulcus (dotted line). $\boldsymbol{b}$, StTA of $A \rightarrow B$ EP before (blue) and after (red) conditioning at current amplitude denoted by black box in $\mathbf{c}$. Diamonds indicate peak and trough used to calculate amplitude plotted in $c$. Inset shows magnified view of the early part of the EP; black lines and arrows indicate the slope of the first response phase. c, Stimulusresponse curve of $A \rightarrow B$ EP before (blue) and after (red) conditioning. Black box is current amplitude, $400 \mu A$ depicted in $\boldsymbol{b}$. Inset, Stimulus-response curve for slope of the first phase with the same color scheme. $\boldsymbol{d}$, Percentage change in EPs from $A, B$, and C to all other sites where there was a response, as in Figure 7. Fifteen EP sites showed statistically significant changes, either increases or decreases, although none was as large as for the $A \rightarrow B$ EP. Significant EPs were $A \rightarrow C, A \rightarrow L 6, A \rightarrow L 8, A \rightarrow L 9, A \rightarrow L 15, B \rightarrow L 1$, $\mathrm{B} \rightarrow \mathrm{C}, \mathrm{B} \rightarrow \mathrm{A}, \mathrm{B} \rightarrow \mathrm{L} 4, \mathrm{~B} \rightarrow \mathrm{L} 10, \mathrm{~B} \rightarrow \mathrm{L} 13, \mathrm{C} \rightarrow \mathrm{A}, \mathrm{C} \rightarrow \mathrm{L} 6, \mathrm{C} \rightarrow \mathrm{B}$, and $\mathrm{C} \rightarrow \mathrm{L} 11$.

EPs were not different from those in the $\mathrm{B}_{\text {cont }}$ distribution (Fig. $10 d ; p=0.27, \mathrm{~K}-\mathrm{S}$ test, lower left quadrant of significance matrix; Fig. 10) regardless of paired-stimulation delay (50-200 $\mathrm{ms}$ delays: $n=6$ sessions, $p=0.26, \mathrm{~K}-\mathrm{S}$ test).

\section{Paired stimulation produces inconsistent STDP effects at most site pairs}

The results from the two site pairs discussed above demonstrated that STDP-like effects could be induced between cortical neurons after paired stimulation. We sought to extend these results to multiple sites and tested 13 other pairs in the hand area of primary motor and somatosensory cortex across both monkeys (Fig.
11) using a $20 \mathrm{~ms}$ interstimulus delay. These sites encompassed both M1 and S1. However, we did not see any significant difference in effects on EP amplitude when considering connections within a particular region $(\mathrm{M} 1 \rightarrow \mathrm{M} 1, \mathrm{~S} 1 \rightarrow \mathrm{S} 1)$ or those that crossed the central sulcus $(\mathrm{M} 1 \rightarrow \mathrm{S} 1, \mathrm{~S} 1 \rightarrow \mathrm{M} 1)$ (one-way ANOVA, $p=0.18)$. The changes in the $\mathrm{A} \rightarrow \mathrm{B}$, $\mathrm{B} \rightarrow \mathrm{A}$, and $\mathrm{A} \rightarrow \mathrm{C}$ connections at $20 \mathrm{~ms}$ delay for all site pairs tested are shown in Figure 12 and Tables 1 and 2 summarize all conditioning experiment parameters. The two site pairs discussed above (Q: $\mathrm{R} 7 \rightarrow \mathrm{R} 11$ and $\mathrm{U}: \mathrm{L} 3 \rightarrow \mathrm{L} 7$, leftmost in Fig. 12) highlight a pattern that is consistent with an STDP time course: an increased $\mathrm{A} \rightarrow \mathrm{B}$ connection and minimal changes in $\mathrm{B} \rightarrow \mathrm{A}$ and $\mathrm{A} \rightarrow \mathrm{C}$. Across all 13 site pairs tested, only one other pair (monkey $\mathrm{Q}: \mathrm{L} 7 \rightarrow \mathrm{L} 11$ ) showed this pattern, but it was unrepeatable in two other sessions (Fig. 12).

To examine the significance of the paired stimulation effects compared with the global effects for all site pairs, we again calculated cumulative distributions. Figure $13 a$ summarizes the results for sessions with a $20 \mathrm{~ms}$ delay for all 15 site pairs as the $\mathrm{A}_{\text {cont }}$ distribution compared with the cumulative density of $\mathrm{A} \rightarrow \mathrm{B}$ and $\mathrm{A} \rightarrow \mathrm{C}$ effects. Similar distributions are shown for the B and C sites (Fig. 13b,c). There was a small, but significant, shift in the mean of $\mathrm{A}_{\text {cont }}(+2.5 \pm 0.9 \%, 1$-sample $t$ test, $p=0.01, n=251$, Fig. $13 a$, inset) and $\mathrm{B}_{\text {cont }}(+2.7 \pm 1.0 \%, p=0.01, n=237$, Fig. 13b, inset) away from zero after conditioning at a 20 ms delay, demonstrating the global changes in connections from stimulated sites. The mean of the $\mathrm{C}_{\text {cont }}$ distribution was not significantly different from zero $(-0.23 \pm 0.28 \%, p=0.68$, Fig. $13 c$, inset), suggesting that the connections from unstimulated sites were not globally affected by conditioning. The $p$-values for these tests are shown in the diagonal elements of the significance matrix shown at the bottom of Figure 13.

The $\mathrm{A} \rightarrow \mathrm{B}$ distribution showed significant separation from the $A_{\text {cont }}$ distribution $(p=0.03, \mathrm{~K}-\mathrm{S}$ test, upper middle element of the significance matrix; Fig. 13a), particularly at large percentage increases (the upper right tail of the $\mathrm{A} \rightarrow \mathrm{B}$ curve), again indicating an effect of targeted conditioning beyond the global effects on the network. Only three of the 15 site pairs tested (Monkey U: L3 $\rightarrow$ L7; Monkey Q: R7 $\rightarrow$ R11 and L7 $\rightarrow$ L11; Fig. 12), including the two pairs described in Figures 7 and 8, drive this separation and lie outside of the $95^{\text {th }}$ percentile of the $\mathrm{A}_{\text {cont }}$ distribution. In contrast, there were no significant differences in the distributions for the $\mathrm{B} \rightarrow \mathrm{A}$ connection $(p=0.08$, middle left element of the significance matrix) and the $\mathrm{B} \rightarrow \mathrm{C}$ connection $(p=0.31)$ compared with $\mathrm{B}_{\text {cont }}$ (Fig. 13b) or for the $\mathrm{C} \rightarrow \mathrm{A}$ connection $(p=0.63)$ and the 
$\mathrm{C} \rightarrow \mathrm{B}$ connection $(p=0.36)$ compared with the $\mathrm{C}_{\text {cont }}$ distribution (Fig. 13c). The $\mathrm{A} \rightarrow \mathrm{C}$ distribution was marginally significantly different from $\mathrm{A}_{\text {cont }}(p=0.05)$.

An example site pair that did not exhibit a significant conditioning effect (Table 2, session 20150708) is shown in Figure 14. The $\mathrm{A} \rightarrow \mathrm{B}$ EP amplitude was $722.6 \pm 6.6 \mu \mathrm{V}$ before conditioning and $697.6 \pm 6.0 \mu \mathrm{V}$ after conditioning $(-3.5 \%, p>0.05$, Fig. $14 b-$ d). Again, we measured the slope of the rising phase of the EP to confirm that the absence of an effect was not due to the peak-trough measure. As can be seen in the insets of Figure $14, b$ and $c$, the changes in slope before and after conditioning closely follow those of the peak-trough amplitude (Fig. 4c, gray filled circle). This was seen consistently across many experiments. There was no significant difference between the percentage change seen in $\mathrm{A} \rightarrow \mathrm{B}$ after conditioning using the peak-trough amplitude compared with the slope of the first rising phase ( $p=0.66$, paired $t$ test; Fig. $4 c$ ) or the absolute amplitude of the first phase ( $p=0.38$, paired $t$ test; Fig. $4 d$ ).

\section{Discussion}

This study aimed to induce synaptic plasticity in sensorimotor cortex in awake, behaving nonhuman primates using a pairedstimulation protocol. Sites in precentral and/or postcentral cortex were deemed functionally connected if stimulation of one site evoked an LFP response at the other site. Prolonged paired stimulation of some connected sites at a fixed delay resulted in STDP, as evidenced by increased EP amplitude when conditioning with short delays but not long ones (Fig. 9). Plasticity effects in the targeted $A \rightarrow B$ connection surpassed global increases in connectivity throughout the network (Fig. 13). Surprisingly, this effect was only produced in two of 15 site pairs (13\%). Effects in the other 13 site pairs predominantly showed global increases in connectivity (50\% of pairs) or depression of the targeted site pair ( $44 \%$ of pairs), likely resulting from changes in excitability or generalized plasticity (Pascual-Leone et al., 1998). These results are consistent with other studies, both in vitro ( $\mathrm{Bi}$ and Poo, 1998) and in vivo (Jackson et al., 2006), showing that the expected effects of STDP were variable and occurred only in a subset of cases. The effects that we observed raise questions about the mechanisms underlying STDP between cortical populations induced with paired electrical stimulation in vivo.

\section{STDP between cortical populations} may be induced via paired stimulation Typical in vitro STDP studies document connectivity changes using a direct measure of EPSPs (Markram et al., 1997; Bi and Poo, 1998; Sjöström et al., 2001; Froemke and Dan, 2002). Evidence of STDP has also been obtained with indirect measures such as evoked movements (Jackson et al., 2006; Lucas and Fetz, 2013), postspike electromyographic retest).

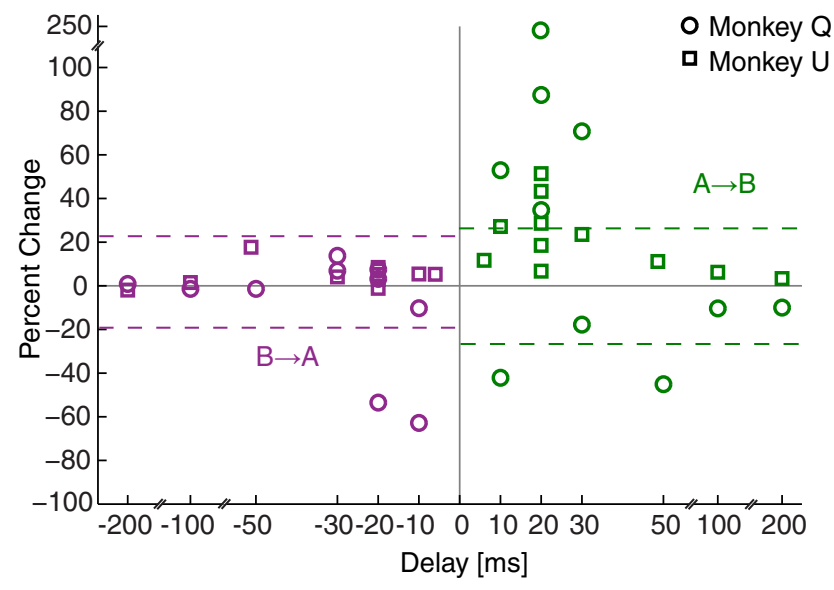

Figure 9. Conditioning effect as a function of paired-stimulation delay. Conditioning delay versus percentage change in EP amplitude immediately after paired stimulation ended between sites shown in Figure 5 (Monkey $Q$, circles) and Figure 8 (Monkey $\mathrm{U}$, squares). Positive delays indicate pre $\rightarrow$ post $(A \rightarrow B)$ stimulation and negative delays indicate post $\rightarrow$ pre $(B \rightarrow A)$ stimulation. Dotted lines show 2.5 th and 97.5 th percentiles for the A distribution (green) or the B distribution (purple) from Figure $10 a$ and $C$, respectively. a

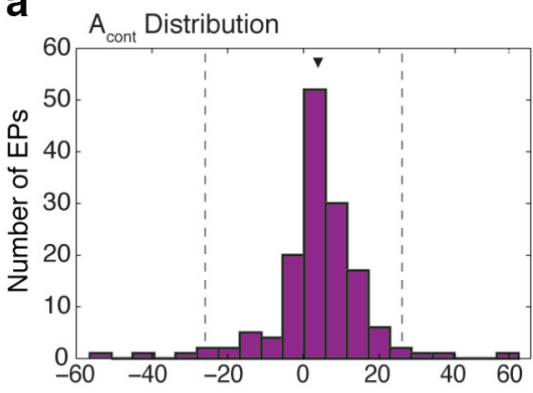

C

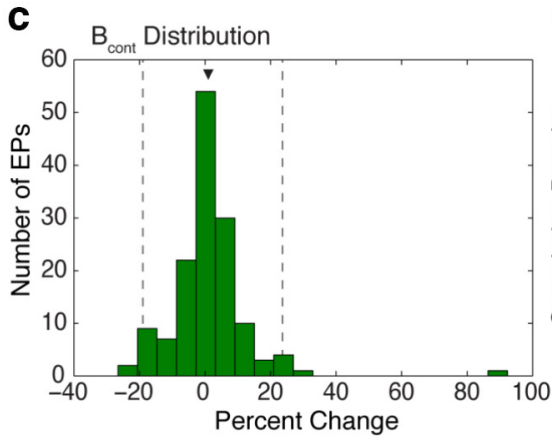

b

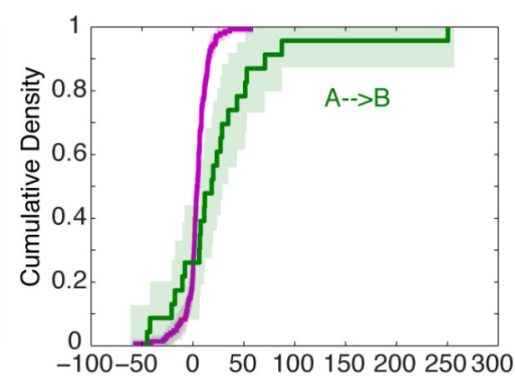

d

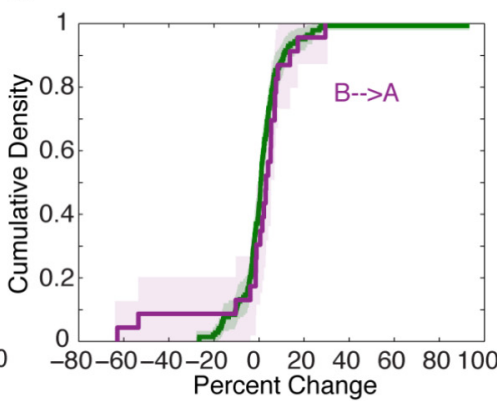

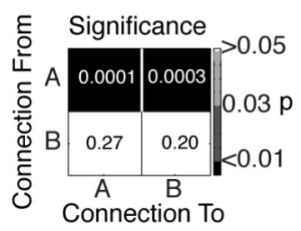

Figure 10. Cumulative density curves describe network-wide effects of conditioning. $\boldsymbol{a}, A_{\text {cont }}$ distribution for all conditioning sessions for the two site pairs depicted in Figures 7 and 8 showing the percentage change in EP amplitude from all sites except $B$ and $C$ for which stimulation at site $A$ evokes a response; arrowhead denotes mean $(+4.0 \%, n=146$ EPs, $p<$ 0.001 ) and dotted lines indicated the 2.5 th and 97.5 th percentiles, which are recapitulated in Figure $9 . \boldsymbol{b}, A_{\text {cont }}$ distribution depicted as a cumulative density (purple) superimposed with the cumulative density of percentage change in $A \rightarrow B E P S$ (green) from all delays depicted in Figure 9. Light-colored shadows indicate the $95 \%$ confidence interval. $c$, $B_{\text {cont }}$ distribution (mean $=+1.3 \%, n=143 \mathrm{EPs}, p=0.2$ ), as in $\boldsymbol{a}$. $\boldsymbol{d}, \mathrm{B}_{\text {cont }}$ distribution cumulative density (green) compared with cumulative density of percentage change in $B \rightarrow A$ EPs (purple), as in $\boldsymbol{b}$. Bottom right, Significance matrix for all effects: on-diagonal quadrants indicate $p$-value of $A_{\text {cont }}$ and $B_{\text {cont }}$ means compared with zero (one-sample $t$ test); off-diagonal quadrants indicate comparison of the directed connection with the control distribution in the same row (two-sample K-S 


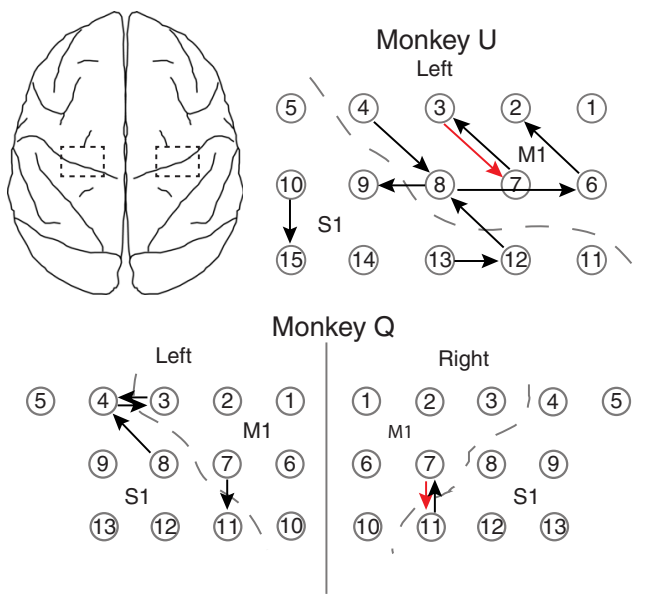

Figure 11. Schematic of all conditioning pairs. Top left, Top-down view of macaque brain; dashed boxes outline positions of implant area bilaterally. Top right, Left electrode grid in Monkey $\mathrm{U}$ with $\mathrm{A} \rightarrow \mathrm{B}$ pairs used for conditioning sessions indicated by arrows; arrow points from A to B. Only the left hemisphere was used in Monkey $U$, which performed a behavioral task using the right hand. Bottom, Bilateral electrode grids for Monkey $Q$ with $A \rightarrow B$ pairs used for conditioning sessions indicated. Dashed lines in Monkeys $\mathrm{U}$ and $\mathrm{Q}$ mark the central sulcus.

ticity under the appropriate circumstances. Surprisingly, a majority of sites did not show STDP, which could be explained by several factors, as discussed below.

\section{STDP is a multifaceted phenomenon}

Paired stimulation of a source population at A and a target population at $\mathrm{B}$ could be considered comparable to the pre$\rightarrow$ post pairing in in vitro studies (Bi and Poo, 1998; Feldman, 2000; Froemke and Dan, 2002). In principle, paired electrical stimulation in vivo will similarly induce activity at the presynaptic and postsynaptic sites in an appropriately timed manner to induce plasticity. However, as has been demonstrated in vitro, timing is only one of many factors governing plasticity at a given synapse (Feldman, 2012). We found that pairs of single pulses were insufficient, but trains of three pulses produced results consistent with observations in rodents (Rebesco and Miller, 2011). Sjöström et al. (2001) demonstrated the influences of background firing rate and cooperativity of multiple inputs on STDP effects in V1 with fixed pre $\rightarrow$ post delays. Spatiotemporal integration is critical for sufficient depolarization in the postsynaptic neuron such that a well-timed back-propagating action potential will promote STDP (Lisman and Spruston, 2005).

The intrinsic connectivity of cortical networks, comprising the number, strength, and location of connections, is another key factor governing synaptic plasticity (Sjöström et al., 2001; Froemke et al., 2005). Recordings of EPs from large populations provide only a gross measure of network connectivity that cannot resolve the specific synapses that might be conditioned. Of the many possible connections between cells at sites A and B, Figure 15 illustrates key pathways that could help to explain our mixed results. Long-range pyramidal cell collaterals identified in neuroanatomical studies (DeFelipe et al., 1986) likely provide the major excitatory pathway giving rise to EPs; these would be modified by conditioning and are probably the site of plastic effects observed in other in vivo STDP studies (Jackson et al., 2006; Rebesco et al., 2010; Rebesco and Miller, 2011). Because LFPs incorporate many nearby current sources (Buzsáki et al., 2012), the EPs cannot differentiate those pathways that may be more amenable to STDP. Because spatial integration is one of the factors contributing to STDP (Sjöström et al., 2001), pathways having high convergence would be

sponses (Nishimura et al., 2013), motor evoked potentials (Wolters et al., 2003), network modeling (Rebesco et al., 2010; Rebesco and Miller, 2011), and others (Feldman, 2012). This study is one of the first to show long-lasting STDP in evoked LFPs mediated by cortical connections. The corticocortical EPs typically showed multiple phases, probably mediated by monosynaptic and polysynaptic connections between the sites. In two of the site pairs, STDP effects were robust, repeatable, and displayed a classic STDP time course (Fig. 9). The changes were evident in multiple EP phases (Fig. $5 c$ ), suggesting that many pathways between the two sites were similarly enhanced by paired stimulation. These results indicate that paired stimulation can induce targeted plas- more likely to show plasticity (Fig. 15, green pathway)

There are also multiple forms of STDP that occur in a regional and synapse-specific fashion (Feldman, 2012). In traditional Hebbian STDP, positive delays (pre before post) produce LTP, whereas negative delays (post before pre) promote LTD. Conversely, in anti-Hebbian STDP, LTD is produced at positive delays and moderate LTP at negative delays. Anti-Hebbian STDP occurs at a variety of synapses, particularly those of layer $2 / 3$ neuron axons on the distal dendrites of layer 5 neurons, likely due to attenuation of the back-propagating action potential (Sjöström and Häusser, 2006). Because our paired stimulation probably invoked STDP at both proximal and distal dendrites, 

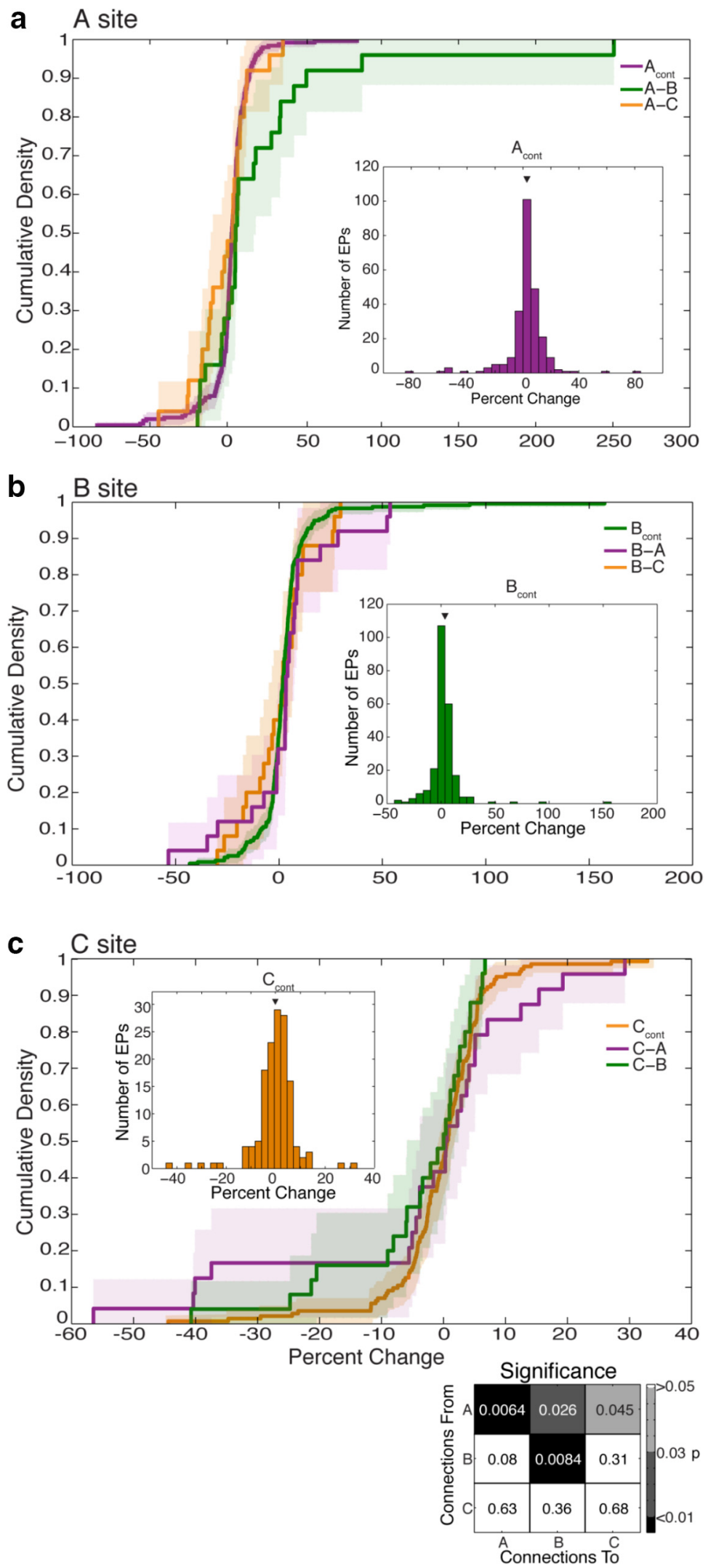

Figure 13. Network-wide effects of paired stimulation across all sessions. $\boldsymbol{a}$, Cumulative densities for the $A_{\text {cont }}$ distribution (purple) from all site pairs at a conditioning delay of 20 ms overlaid with the $A \rightarrow B$ (green) and $A \rightarrow C$ (orange) distributions. $\boldsymbol{b}$, Cumulative densities for the $B_{\text {cont }}$ (green curve), $B \rightarrow A$ (purple), and $B \rightarrow C$ (orange) distributions. $c$, Cumulative densities for the $C_{\text {cont }}$ (orange curve), $C \rightarrow A$ (purple), and $C \rightarrow B$ (green) distributions. For all panels, light-colored shadow indicates $95 \%$ confidence interval; insets show histograms for the appropriate control distribution and arrowheads mark the mean. Cumulative densities were calculated similarly to those in Figure 10. Bottom right, Significance matrix for all combinations: on-diagonal the resulting mixture of Hebbian and anti-Hebbian effects could have prevented net LTP from occurring at site B (Fig. 15, purple pathway). Connections between excitatory and inhibitory neurons (Fig. 15, red pathway) show a variety of STDP time courses (Caporale and Dan, 2008), further obscuring the net effects produced among a heterogeneous population. It should also be noted that fields from antidromically activated cells at B would presumably not be changed by the conditioning protocol and thus would dilute the measure of any actual $\mathrm{A} \rightarrow \mathrm{B}$ conditioning.

Traditional Hebbian STDP also predicts depression in the $\mathrm{B} \rightarrow \mathrm{A}$ connections because this involves a negative delay. To the extent that the $\mathrm{B} \rightarrow \mathrm{A}$ connections from distal layer $2 / 3$ at $B$ to layer 5 cells at A exhibit anti-Hebbian STDP this would be consistent with the lack of $\mathrm{B} \rightarrow \mathrm{A}$ depression that we observed (Fig. 15, blue pathway). In addition, STDP effects incorporating multiple spike times do not add linearly (Froemke and Dan, 2002) and therefore, whereas $\mathrm{B} \rightarrow \mathrm{A}$ connections may have experienced a negative delay between the initial paired-stimulation pulses, continued activation via recurrence may have interfered with the simplest Hebbian mechanism.

Timing of presynaptic and postsynaptic activation is one of the few parameters that we could control experimentally, along with rate of stimulation. For two of our site pairs, a sufficient combination of these factors aligned such that the rate and timing of paired stimulation promoted STDP. We tested other protocols varying the rate, number of pulses, or duration of stimulation to optimize the parameter space (see Materials and Methods), but these did not produce conditioning effects for the other 13 site pairs. However, it seems likely that the synaptic connections mediating the EPs between these other pairs would be modifiable and that other, untested stimulation protocols would have activated the appropriate circuit elements for inducing STDP at these sites.

\section{Artificial effects of electrical stimulation}

Electrical stimulation is an excellent way to activate neurons rapidly, yet it is inher-

$\leftarrow$

quadrants indicate $p$-value of $A_{\text {cont, }} B_{\text {cont }}$, and $C_{\text {cont }}$ means compared with zero (one-sample $t$ test); off-diagonal quadrants indicate comparison of the directed connection with the control distribution in the same row (two-sample K-S test). 
a

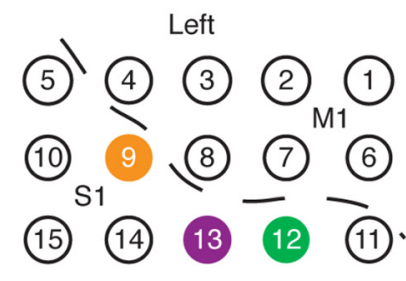

b

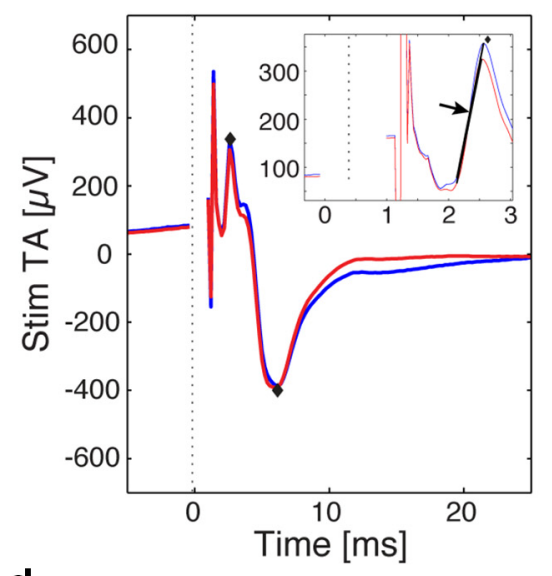

d

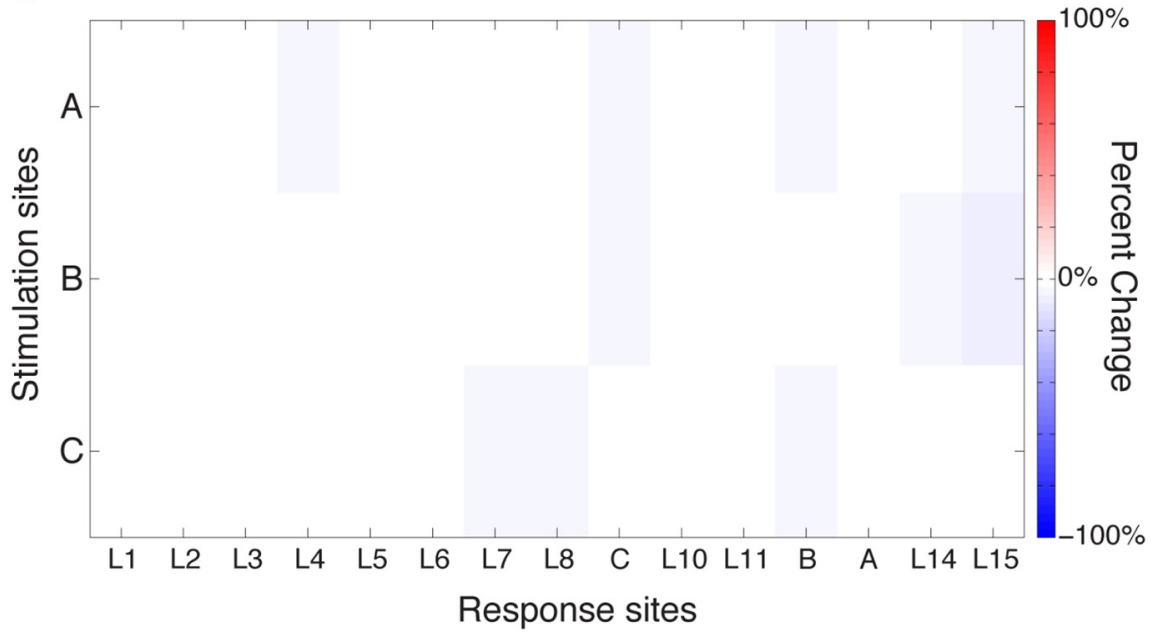

Figure 14. Paired stimulation does not produce a conditioning effect at all sites. $\boldsymbol{a}$, Schematic of recording grid with $A$ (purple), $B$ (green), and C (orange) sites denoted for a conditioning session at a $20 \mathrm{~ms}$ delay in Monkey $\mathrm{U}$ while in the recording booth for $1 \mathrm{~h}$. $\boldsymbol{b}$, StTA of $A \rightarrow B$ EP before (blue) and after (red) conditioning. Inset, Expansion of StTA showing the slope (black line and arrow) of the first EP phase. Black diamonds denote peak and trough used to calculate amplitude in $c$. $c, A \rightarrow B$ stimulus-response curve before (blue) and after (red) conditioning. Black box denotes current depicted in $\boldsymbol{b}$. Inset, Stimulus-response curve before and after conditioning for slope of first EP phase. $\boldsymbol{d}$, Percentage change in EP amplitude from A-C to all other recording sites after conditioning.

ently artificial and nonspecific because it can activate axons and recruit neurons transynaptically (Gustafsson and Jankowska, 1976; Histed et al., 2009) in nonphysiological spatiotemporal patterns. Many studies using electrical stimulation to induce STDP record a neural signal from the presynaptic and postsynaptic site (Markram et al., 1997; Bi and Poo, 1998) or trigger postsynaptic stimulation from a neural event (Jackson et al., 2006; Rebesco et al., 2010; Nishimura et al., 2013; Zanos, 2013) to ensure proper timing of action potentials and postsynaptic depolarization. We hypothesized that paired stimulation would be sufficient to induce well-timed spiking both presynaptically and postsynaptically with fixed delays appropriate to promote plasticity. This hypothesis is substantiated by in vivo evidence that paired stimulation can change the inferred connectivity of neural networks (Rebesco and Miller, 2011). Furthermore, studies in humans using transcranial magnetic stimulation to pair stimulation of the median nerve with associated cortical areas demonstrate classic Hebbian STDP (Wolters et al., 2003). Therefore paired-stimulation protocols should be able to promote targeted plasticity, as we observed for a subset of site pairs. However, it is possible that the artificial pattern of neural activation with electrical stimulation was not permissive for STDP at the other sites.

Conditioning with spike-triggered stimulation (Jackson et al., 2006) produced a larger proportion of positive plasticity effects than paired electrical stimulation, which could be due to several significant experimental differences. Stimulating at site A rather than using recorded trigger spikes would have activated a larger population of more diverse cell types synchronously and consequently recruited a broader range of plasticity mechanisms, such as Hebbian and anti-Hebbian effects. The net result of this broader recruitment may have been less facilitation of excitatory connections, producing fewer cases of significant changes in EPs in the present study. Second, the triggering spikes in the Jackson et al. (2006) study occurred in association with normal behavior, whereas we delivered paired stimulation in a preprogrammed manner independently of the modulation of local activity with movements or sleep spindles. Third, documenting the conditioning effects with peripheral responses to trains of cortical microstimuli rather than with cortical potentials evoked by single test stimuli would have involved different circuit mechanisms and potential loci of plasticity. These issues could be investigated in future experiments by direct comparison of results with spike- and stimulustriggered conditioning.

The complexity of different STDP mechanisms for different cell pairs (Sjöström et al., 2001; Feldman, 2012) could be addressed with more specific stimulation techniques. For example, optogenetic stimulation can be used to activate only local excitatory pyramidal neurons, rather than the diverse, more widely distributed, excitatory and inhibitory population recruited by electrical stimuli (Yazdan-Shahmorad et al., 2016). In a paired-stimulation protocol, optogenetic stimulation could begin to elucidate how different circuit elements contribute to STDP in cortical networks.

In conclusion, our results indicate that conditioning in monkey sensorimotor cortex with paired electrical stimulation produced results consistent with STDP, but in a surprisingly small proportion of sites. The neural mechanisms explaining these results remain to be further elucidated. Future experiments could 


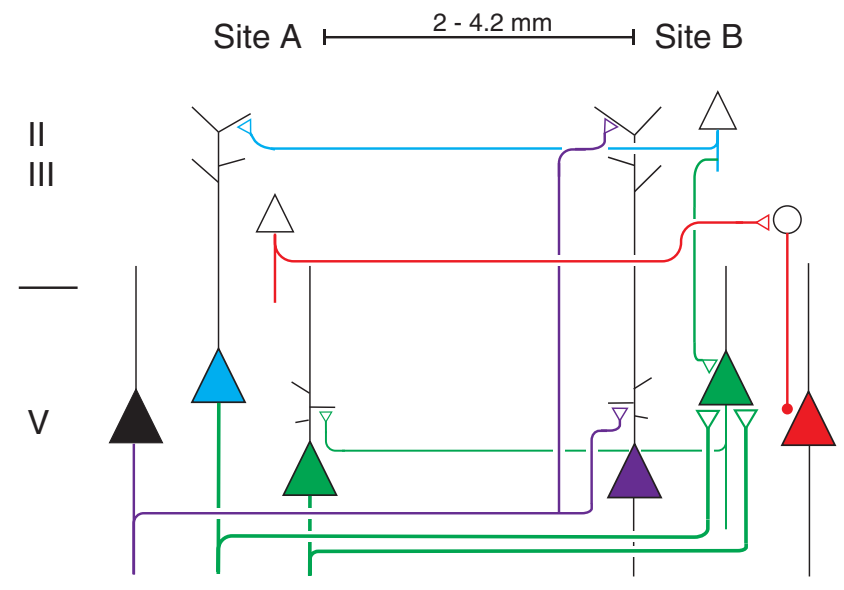

Figure 15. Proposed diagram of interareal connections and STDP effects. Four of many potential pathways connecting an $A$ and $B$ site in sensorimotor cortex are shown to illustrate differential STDP effects produced in these networks and how they may interact. Upper layer $2 / 3$ and layer 5 are the likely targets of the dual electrodes for each site, so only these layers are shown. The green pathway from $A \rightarrow B$ highlights successful potentiation due to convergent excitatory input between layer 5 pyramidal cells, as well as collaterals within the B site. Similarly, the green pathway from $B \rightarrow$ A shows successful depression. The other three pathways do not promote STDP for various reasons. The red pathway results in inhibition between $A$ and $B$ via inhibitory interneurons (circles). In the purple pathway, whereas inputs to the proximal dendrites would promote potentiation, inputs on the distal dendrites would not. Similarly, depression in the blue pathway is unsuccessful because the primary connection from $B \rightarrow A$ is in the distal dendrites.

compare the results of conditioning with electrical stimulation and results with optogenetic or spike-triggered stimulation at the same cortical sites.

\section{References}

Arai N, Müller-Dahlhaus F, Murakami T, Bliem B, Lu MK, Ugawa Y, Ziemann U (2011) State-dependent and timing-dependent bidirectional associative plasticity in the human SMA-M1 network. J Neurosci 31: 15376-15383. CrossRef Medline

Bi GQ, Poo MM (1998) Synaptic modifications in cultured hippocampal neurons: dependence on spike timing, synaptic strength, and postsynaptic cell type. J Neurosci 18:10464-10472. Medline

Bi G, Poo M (2001) Synaptic modification by correlated activity: Hebb's postulate revisited. Annu Rev Neurosci 24:139-166. CrossRef Medline

Brown TH, Kairiss EW, Keenan CL (1990) Hebbian synapses: Biophysical mechanisms and algorithms. Annu Rev Neurosci 13:475-511. CrossRef Medline

Buetefisch C, Heger R, Schicks W, Seitz R, Netz J (2011) Hebbian-type stimulation during robot-assisted training in patients with stroke. Neurorehabil Neural Repair 25:645-655. CrossRef Medline

Bunday KL, Perez MA (2012) Motor recovery after spinal cord injury enhanced by strengthening corticospinal synaptic transmission. Curr Biol 22:2355-2361. CrossRef Medline

Buzsáki G, Anastassiou CA, Koch C (2012) The origin of extracellular fields and currents-EEG, ECoG, LFP and spikes. Nat Rev Neurosci 13:407-420. CrossRef Medline

Caporale N, Dan Y (2008) Spike timing-dependent plasticity: a Hebbian learning rule. Annu Rev Neurosci 31:25-46. CrossRef Medline

DeFelipe J, Conley M, Jones EG (1986) Long-range focal collateralization of axons arising from corticocortical cells in monkey sensory-motor cortex. J Neurosci 6:3749-3766. Medline

Diesmann M, Gewaltig MO, Aertsen A (1999) Stable propagation of synchronous spiking in cortical neural networks. Nature 402:529533.

Feldman DE (2000) Timing-based LTP and LTD at vertical inputs to layer II/III pyramidal cells in rat barrel cortex. Neuron 27:45-56. CrossRef Medline

Feldman DE (2012) The spike-timing dependence of plasticity. Neuron 75: 556-571. CrossRef Medline
Fetz EE, Cheney PD (1980) Postspike facilitation of forelimb muscle activity by primate corticomotoneuronal cells. J Neurophysiol 44:751-772. Medline

Froemke RC, Dan Y (2002) Spike-timing-dependent synaptic modification induced by natural spike trains. Nature 416:433-438. CrossRef Medline

Froemke RC, Poo MM, Dan Y (2005) Spike-timing-dependent synaptic plasticity depends on dendritic location. Nature 434:221-225. CrossRef Medline

Guggenmos DJ, Azin M, Barbay S, Mahnken JD, Dunham C, Mohseni P, Nudo RJ (2013) Restoration of function after brain damage using a neural prosthesis. Proc Natl Acad Sci U S A 110:21177-21182. CrossRef Medline

Gustafsson B, Jankowska E (1976) Direct and indirect activation of nerve cells by electrical pulses applied extracellularly. J Physiol 258:33-61. CrossRef Medline

Hebb DO (1949) The organization of behavior: a neuropsychological theory. New York: Wiley.

Hess G, Aizenman CD, Donoghue JP (1996) Conditions for the induction of long-term potentiation in layer II/III horizontal connections of the rat motor cortex. J Neurophysiol 75:1765-1778. Medline

Histed MH, Bonin V, Reid RC (2009) Direct activation of sparse, distributed populations of cortical neurons by electrical microstimulation. Neuron 63:508-522. CrossRef Medline

Jackson A, Mavoori J, Fetz EE (2006) Long-term motor cortex plasticity induced by an electronic neural implant. Nature 444:56-60. CrossRef Medline

Koch G, Ponzo V, Di Lorenzo F, Caltagirone C, Veniero D (2013) Hebbian and anti-Hebbian spike-timing-dependent plasticity of human corticocortical connections. J Neurosci 33:9725-9733. CrossRef Medline

Lisman J, Spruston N (2005) Postsynaptic depolarization requirements for LTP and LTD: a critique of spike timing-dependent plasticity. Nat Neurosci 8:839-841. CrossRef Medline

Lu JT, Li CY, Zhao JP, Poo MM, Zhang XH (2007) Spike-timingdependent plasticity of neocortical excitatory synapses on inhibitory interneurons depends on target cell type. J Neurosci 27:9711-9720. CrossRef Medline

Lucas TH, Fetz EE (2013) Myo-cortical crossed feedback reorganizes primate motor cortex output. J Neurosci 33:5261-5274. CrossRef Medline

Markram H, Lübke J, Frotscher M, Sakmann B (1997) Regulation of synaptic efficacy by coincidence of postsynaptic APs and EPSPs. Science 275: 213-215. CrossRef Medline

McCarthy G, Wood CC, Allison T (1991) Cortical somatosensory evoked potentials. I. Recordings in the monkey Macaca fascicularis. J Neurophysiol 66:53-63. Medline

McCreery DB, Bullara LA, Agnew WF (1986) Neuronal activity evoked by chronically implanted intracortical microelectrodes. Exp Neurol 92:147161. CrossRef Medline

McPherson JG, Miller RR, Perlmutter SI (2015) Targeted, activity-dependent spinal stimulation produces long-lasting motor recovery in chronic cervical spinal cord injury. Proc Natl Acad Sci U S A 112:12193-12198. CrossRef Medline

Nishimura Y, Perlmutter SI, Eaton RW, Fetz EE (2013) Spike-timingdependent plasticity in primate corticospinal connections induced during free behavior. Neuron 80:1301-1309. CrossRef Medline

Pascual-Leone A, Tormos JM, Keenan J, Tarazona F, Cañete C, Catalá MD (1998) Study and modulation of human cortical excitability with transcranial magnetic stimulation. J Clin Neurophysiol 15:333-343. CrossRef Medline

Rebesco JM, Miller LE (2011) Enhanced detection threshold for in vivo cortical stimulation produced by Hebbian conditioning. J Neural Eng 8:016011. CrossRef Medline

Rebesco JM, Stevenson IH, Körding KP, Solla SA, Miller LE (2010) Rewiring neural interactions by micro-stimulation. Front Syst Neurosci 4: pii: 39. CrossRef Medline

Richardson AG, Fetz EE (2012) Brain state-dependence of electrically evoked potentials monitored with head-mounted electronics. IEEE Trans Neural Syst Rehabil Eng 20:756-761. CrossRef Medline 
Sanes JN, Donoghue JP (2000) Plasticity and primary motor cortex. Annu Rev Neurosci 23:393-415. CrossRef Medline

Sjöström PJ, Häusser M (2006) A cooperative switch determines the sign of synaptic plasticity in distal dendrites of neocortical pyramidal neurons. Neuron 51:227-238. CrossRef Medline

Sjöström PJ, Turrigiano GG, Nelson SB (2001) Rate, timing, and cooperativity jointly determine cortical synaptic plasticity. Neuron 32:11491164. CrossRef Medline

Stuchlik A (2014) Dynamic learning and memory, synaptic plasticity and neurogenesis: an update. Front Behav Neurosci 8:106. CrossRef Medline

Wolters A, Sandbrink F, Schlottmann A, Kunesch E, Stefan K, Cohen LG, Benecke R, Classen J (2003) A temporally asymmetric Hebbian rule governing plasticity in the human motor cortex. J Neurophysiol 89:23392345. CrossRef Medline

Yazdan-Shahmorad A, Diaz-Botia C, Hanson TL, Kharazia V, Ledochowitsch P, Maharbiz MM, Sabes PN (2016) A large-scale interface for optogenetic stimulation and recording in nonhuman primates. Neuron 89:927939. CrossRef Medline

Zanos S (2013) Cortical surface recurrent brain-computer interfaces. Doctoral dissertation. University of Washington, Seattle, WA.

Zanos S, Richardson AG, Shupe L, Miles FP, Fetz EE (2011) The Neurochip-2: an autonomous head-fixed computer for recording and stimulating in freely behaving monkeys. IEEE Trans Neural Syst Rehabil Eng 19:427-435. CrossRef Medline 